\title{
A multiscattering series for impedance tomography in layered media
}

\author{
Liliana Borcea $\nmid \S$ and Michael Ortiz $\ddagger \|$ \\ $\dagger$ Computational and Applied Mathematics, MS 134, Rice University, 6100 Main Street, \\ Houston, TX 77005-1892, USA \\ ‡ Graduate Aeronautical Laboratories 105-50, California Institute of Technology, Pasadena, CA \\ 91125, USA
}

Received 19 May 1998

\begin{abstract}
We introduce an inversion algorithm for tomographic images of layered media. The algorithm is based on a multiscattering series expansion of the Green function that, unlike the Born series, converges unconditionally. Our inversion algorithm obtains images of the medium that improves iteratively as we use more and more terms in the multiscattering series. We present the derivation of the multiscattering series, formulate the inversion algorithm and demonstrate its performance through numerical experiments.
\end{abstract}

\section{Introduction}

We consider the inverse problem of imaging the electrical conductivity of a layered medium occupying the half-space $\Omega=\{(x, y, z) \mid z \leqslant=0\}$. The data are the applied electric currents and the measured electric voltages on the boundary surface $S=\{(x, y, z) \mid z=0\}$. This work is motivated by problems arising in geophysical prospecting, where, in many situations, it is reasonable to approximate the Earth by a layered medium in the half-space $\Omega$. The upper half space is assumed to be composed of dry air with negligible electrical conductivity.

The electric potential $\phi$ satisfies

$$
\begin{aligned}
& \nabla \cdot[\sigma(z) \nabla \phi(x, y, z)]=0 \quad \text { in } \Omega \\
& \sigma(0) \frac{\partial \phi}{\partial z}(x, y, 0)=j(x, y) \quad \text { on } S \\
& \phi(x, y, z) \rightarrow 0 \quad \text { as } \quad z \rightarrow-\infty \\
& \phi, \quad \frac{\partial \phi}{\partial x}, \quad \frac{\partial \phi}{\partial y} \text { and } \quad j \rightarrow 0 \quad \text { as } \quad x, y \rightarrow \pm \infty,
\end{aligned}
$$

where $\sigma$ is the electrical conductivity which depends only on depth and $j$ is the given normal current at the boundary. For problem (1.1) to be well posed, we must have

$$
\int_{S} j(x, y) \mathrm{d} x \mathrm{~d} y=0 .
$$

The conductivity $\sigma$ is a real, positive function and the current $j$ is a function in the space $H^{-\frac{1}{2}}(S)$. We overspecify the problem by requiring that

$$
\phi(x, y, 0)=v(x, y) \quad \text { on } S,
$$

$\S$ E-mail address: borcea@caam.rice.edu

|| E-mail address: ortiz@madrid.caltech.edu 


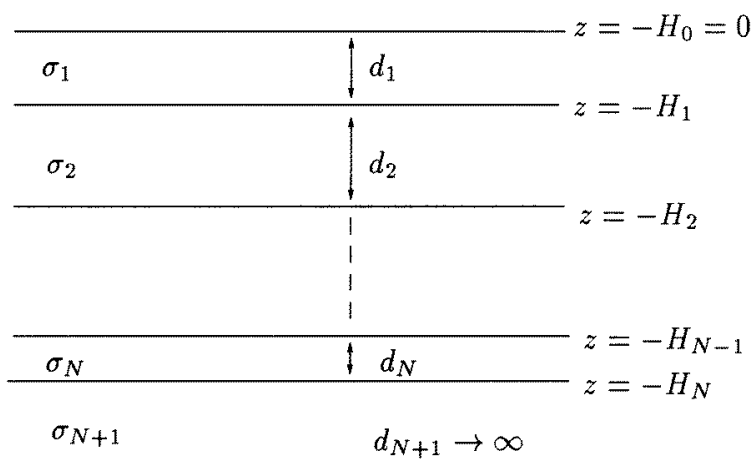

Figure 1. Discrete layered medium.

where $v$ is the measured voltage. The inverse problem is to find $\sigma(z)$ for $z<0$ from the Neumann to Dirichlet map

$$
\Gamma: j \rightarrow v, \quad \text { where } \quad \Gamma: H^{-\frac{1}{2}}(S) \rightarrow H^{\frac{1}{2}}(S) .
$$

In practice, $j$ and $v$ are not known over the whole boundary $S$ but rather at discrete locations confined in a bounded subdomain $\mathcal{M} \subset S$. Thus, the imaging of $\sigma(z)$ is to be done with only partial knowledge of the Neumann to Dirichlet map (1.5).

We assume a discrete layered medium as shown in figure 1 . The case of a continuous $\sigma(z)$ can be viewed as the limit of the discrete case, where the width of the layers is infinitesimally small. Under the assumption of a discontinuous electrical conductivity, suppose that we have $N+1$ layers of width $d_{i}$, where $i=1, \ldots N+1$. The last layer is assumed infinite, so $d_{N+1} \rightarrow \infty$. In each layer, the conductivity is given by

$\sigma(z)=\sigma_{i}=$ constant,$\quad$ for $-H_{i}<z \leqslant-H_{i-1}, i=1, \ldots N+1$.

The forward problem (1.1) for a layered medium with conductivity (1.6) can be solved explicitly (see for example $[21,25,26])$ and the electric potential on the boundary $S$ is

$$
\begin{aligned}
\phi(x, y, 0) & =v(x, y) \\
& =\frac{1}{2 \pi} \int_{-\infty}^{\infty} \int_{-\infty}^{\infty} \frac{J(\boldsymbol{k})}{|\boldsymbol{k}| \sigma_{1}}\left[\frac{1+R(|\boldsymbol{k}|, \boldsymbol{\sigma}, \boldsymbol{d}) \mathrm{e}^{-2|\boldsymbol{k}| d_{1}}}{1-R(|\boldsymbol{k}|, \boldsymbol{\sigma}, \boldsymbol{d}) \mathrm{e}^{-2|\boldsymbol{k}| d_{1}}}\right] \exp [-\mathrm{i} \boldsymbol{k} \cdot(x, y)] \mathrm{d} \boldsymbol{k},
\end{aligned}
$$

where $J(k)$ is the Fourier transform of the boundary current $j, k$ is the Fourier frequency, $\boldsymbol{\sigma}=\left(\sigma_{1}, \ldots \sigma_{N+1}\right)$ and $\boldsymbol{d}=\left(d_{1}, \ldots d_{N+1}\right)$. The kernel $R(|\boldsymbol{k}|, \boldsymbol{\sigma}, \boldsymbol{d})$ is given by

$$
R(|k|, \sigma, d)=r_{1},
$$

where $r_{i}, i=1, \ldots N$ are calculated iteratively, starting from the bottom layer:

$$
\begin{aligned}
& r_{N+1}=0 \\
& r_{n}=\frac{r_{n+1}+D_{n} \mathrm{e}^{2|k| d_{n+1}}}{\mathrm{e}^{2|k| d_{n+1}}+D_{n} r_{n+1}} \\
& D_{n}=\frac{\sigma_{n}-\sigma_{n+1}}{\sigma_{n}+\sigma_{n+1}}, \quad \text { for } n=1, \ldots N .
\end{aligned}
$$

Equation (1.7) clearly shows the nonlinearity of the inverse problem, where the kernel $R$ and the measured voltage $v$ have a complicated dependence on the conductivity $\sigma(z)$. 
One approach to solving the inverse problem is layer stripping, based on a Ricatti equation for $r(z)$ :

$$
\begin{aligned}
& r^{\prime}(z)+2|\boldsymbol{k}| r(z)-\frac{1}{2} \frac{\mathrm{d} \ln \sigma(z)}{\mathrm{d} z}\left[1-r^{2}(z)\right]=0 \\
& r(z) \rightarrow 0 \quad \text { as } z \rightarrow-\infty
\end{aligned}
$$

that can be obtained from the discrete equations (1.9) applied to a continuum by setting $r\left(-H_{n}\right)=r_{n}$ and taking the limit $d_{i} \rightarrow 0, i=1, \ldots N$ as $N \rightarrow \infty$. Another proof of (1.10) follows from equation (1.1) after taking the Fourier transform with respect to $x$ and $y$. We have

$$
\begin{aligned}
& \frac{\partial}{\partial z}\left[\sigma(z) \frac{\partial \hat{\phi}}{\partial z}(\boldsymbol{k}, z)\right]-|\boldsymbol{k}|^{2} \sigma(z) \hat{\phi}(\boldsymbol{k}, z)=0 \quad \text { for } z<0 \\
& \frac{\partial \hat{\phi}}{\partial z}(\boldsymbol{k}, 0)=\frac{J(\boldsymbol{k})}{|\boldsymbol{k}| \sigma_{1}} \\
& \hat{\phi}(\boldsymbol{k}, z) \rightarrow 0 \quad \text { as } \quad z \rightarrow-\infty
\end{aligned}
$$

and we define

$$
r(z)=\left[1-\frac{\partial \hat{\phi}(\boldsymbol{k}, z) / \partial z}{|\boldsymbol{k}| \hat{\phi}(\boldsymbol{k}, z)}\right] /\left[1+\frac{\partial \hat{\phi}(\boldsymbol{k}, z) / \partial z}{|\boldsymbol{k}| \hat{\phi}(\boldsymbol{k}, z)}\right] .
$$

Since the solution of interest satisfies $\hat{\phi}(\boldsymbol{k}, z) \sim \mathrm{e}^{|\boldsymbol{k}| z}$ as $z \rightarrow-\infty, r(z)$ defined by (1.12) satisfies the decay condition at infinity. Furthermore, $r(z)$ satisfies the Ricatti equation (1.10) as one can easily check by direct substitution. Layer stripping has been explored in inverse scattering problems for the Helmholtz equation [11,30]. The idea of layer stripping based on a different Ricatti equation than (1.10) has been used in electric impedance tomography in [29]. Furthermore, the method has been extended to imaging a rectangular resistor network in [14]. The main problem of these algorithms is the stability issue for the high-frequency modes.

In this paper, we propose another approach to solving the inverse problem (1.1)-(1.4). We construct a multiscattering series expansion of the kernel $R(|\boldsymbol{k}|, \boldsymbol{\sigma}, \boldsymbol{d})$ in (1.7). The series is in powers of the reflection coefficients $D_{n}=\frac{\sigma_{n}-\sigma_{n+1}}{\sigma_{n}+\sigma_{n+1}}$ and, unlike the Born series, converges for all contrasts. The series expansion of the kernel $R$ is obtained through a calculation of the Green function of (1.1) via path integration [16, 20, 28, 32]. In section 2 we explain the derivation of the multiscattering series via path integration. In section 3 we introduce the inversion algorithm. Numerical results are presented in section 4.

\section{Derivation of the multiscattering series}

\subsection{Path-integral representation of the Green function}

We begin the derivation of the path integral representation of the Green function by considering the elliptic problem for the whole space

$$
\begin{array}{ll}
\nabla \cdot\left[\sigma(\boldsymbol{x}) \nabla G\left(\boldsymbol{x}, \boldsymbol{x}^{\prime}\right)\right]=-\delta\left(\boldsymbol{x}-\boldsymbol{x}^{\prime}\right), & \text { for } \boldsymbol{x}, \boldsymbol{x}^{\prime} \in \mathbb{R}^{3} \\
G\left(\boldsymbol{x}, \boldsymbol{x}^{\prime}\right) \rightarrow 0 & \text { as } \quad\left|\boldsymbol{x}-\boldsymbol{x}^{\prime}\right| \rightarrow \infty
\end{array}
$$

However, we explain later how to reduce the domain to the half-space $\Omega$, by introducing a special extension of $\sigma(x)$ for $z>0$. Suppose that we discretize (2.1) by reducing the space to a cubic infinite lattice of spacing $h$. The discretization of equation (2.1) leads to an algebraic system of equations:

$$
\sum_{b \in \Lambda} K_{a b} u_{b}=f_{a}
$$




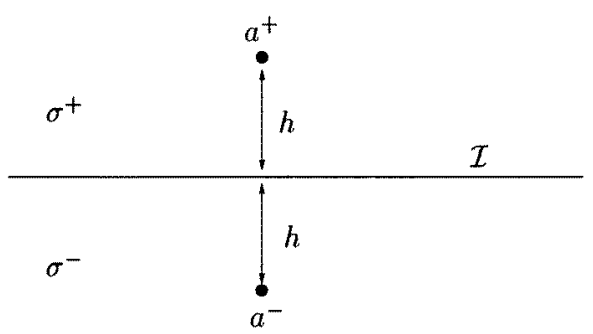

Figure 2. Interface $\mathcal{I}$ between two layers of different electrical conductivity.

where $\Lambda$ is the set of indices of the nodes in the lattice, $u_{b}=G\left(\boldsymbol{x}_{b}, \boldsymbol{x}^{\prime}\right)$ and $f_{a}=\delta\left(\boldsymbol{x}_{a}-\boldsymbol{x}^{\prime}\right)$. The coefficients $K_{a b}$ satisfy

$$
\begin{aligned}
& K_{a b}=K_{b a} \leqslant 0 \quad \text { for } \quad a \neq b ; \quad a, b \in \Lambda \\
& K_{a a}>0, \quad \text { for all } a \in \Lambda \\
& \sum_{b \in \Lambda} K_{a b}=0, \quad \text { for all } a \in \Lambda .
\end{aligned}
$$

As an example of discretization of (2.1) in a region with constant $\sigma$, consider central differences:

$$
\frac{\sigma_{a}}{h^{2}}\left[6 u_{a}-\sum_{b \in \mathcal{N}(a)} u_{b}\right]=f_{a},
$$

where $\mathcal{N}(a)=\left\{b \in \Lambda|| \boldsymbol{x}_{b}-\boldsymbol{x}_{a} \mid=h\right\}$ is the set of neighbours of the node $a$ and $\sigma_{a}=\sigma\left(\boldsymbol{x}_{a}\right)$. For a node $a \in \Lambda$ that belongs to an interface $\mathcal{I}$ between two layers (see figure 2), the discretization of (2.1) is

$$
\frac{\langle\sigma\rangle}{h^{2}}\left[6 u_{a}-\sum_{b \in \mathcal{N}_{\mathcal{I}}(a)} u_{b}-(1+D) u_{a^{+}}-T u_{a^{-}}\right]=f_{a},
$$

where

$$
\begin{aligned}
& \langle\sigma\rangle=\frac{\sigma^{+}+\sigma^{-}}{2}, \\
& D=\frac{\sigma^{+}-\sigma^{-}}{\sigma^{+}+\sigma^{-}}=\text {reflection coefficient, } \\
& T=\frac{2 \sigma^{-}}{\sigma^{+}+\sigma^{-}}=1-D=\text { transmission coefficient }
\end{aligned}
$$

and $\mathcal{N}_{\mathcal{I}}(a)=\left\{b \in \Lambda|| x_{b}-x_{a} \mid=h\right.$, and $\left.x_{b} \in \mathcal{I}\right\}$ is the set of neighbours of node $a$, that belong to the interface.

Based on the coefficients $K_{a b}$ we define a new set of coefficients

$$
\begin{aligned}
& \tau_{x_{a}, x_{b}}=-\frac{K_{a b}}{K_{a a}} \geqslant 0, \quad \text { for } \quad a \neq b ; a, b \in \Lambda \\
& \sum_{b \neq a} \tau_{x_{a}, x_{b}}=1,
\end{aligned}
$$

which are given the interpretation of transition probability from node $a$ to node $b$ in the lattice. Next, we solve the linear system (2.2) with Jacobi's iterative method:

$$
\begin{aligned}
u_{a}^{(0)} & =\frac{f_{a}}{K_{a a}} \\
u_{a}^{(n)} & =\frac{f_{a}}{K_{a a}}+\sum_{b \neq a} \tau_{x_{a}, x_{b}} u_{b}^{(n-1)}, \quad \text { for } \quad n=1,2, \ldots
\end{aligned}
$$


and take the limit $h \rightarrow 0, n \rightarrow \infty$ to get the Green function as

$$
G\left(\boldsymbol{x}, \boldsymbol{x}^{\prime}\right)=\lim _{h \rightarrow 0} \sum_{n=0}^{\infty} g_{n}\left(\boldsymbol{x}, \boldsymbol{x}^{\prime}\right) .
$$

In (2.9), $g_{n}\left(\boldsymbol{x}, \boldsymbol{x}^{\prime}\right)$ is the transition probability from point $\boldsymbol{x}^{\prime}$ to point $\boldsymbol{x}$ in $n$ steps, given by

$$
g_{n}\left(\boldsymbol{x}, \boldsymbol{x}^{\prime}\right)=\frac{h^{2}}{6 \sigma(\boldsymbol{x})} \sum_{\Upsilon} \mathcal{P}(\Upsilon \mid n),
$$

where

$$
\mathcal{P}(\Upsilon \mid n)=\prod_{i=1}^{n} \tau_{x_{i-1}, x_{i}}, \quad \boldsymbol{x}_{0}=\boldsymbol{x}^{\prime}, \quad \boldsymbol{x}_{n}=\boldsymbol{x}
$$

is the probability of a path $\Upsilon$ that starts at $\boldsymbol{x}^{\prime}$ and ends at $\boldsymbol{x}$ after $n$ steps. Thus, the Green function has a path integral representation given by (2.9)-(2.11). Furthermore, problem (2.1) has been reduced to a random walk in $\mathbb{R}^{3}$.

\subsection{Isotropic, homogeneous media}

In isotropic, homogeneous media, the transition probabilities $\tau$ can be modelled by a Gaussian [10]

$$
\tau_{x_{i-1}, x_{i}}=\tau\left(\left|\boldsymbol{x}_{i}-\boldsymbol{x}_{i-1}\right|\right)=\left(\frac{3}{2 \pi h^{2}}\right)^{\frac{3}{2}} \exp \left(-\frac{3}{2 h^{2}}\left|\boldsymbol{x}_{i}-\boldsymbol{x}_{i-1}\right|^{2}\right) .
$$

The problem of random flights has an elegant solution due to Markoff $[10,20]$. However, here we give a different calculation that clarifies future steps in the derivation of the multiscattering series of the Green function.

Equations (2.10)-(2.12) give

$g_{n}\left(\boldsymbol{x}, \boldsymbol{x}^{\prime}\right)=\frac{h^{2}}{6 \sigma}\left(\frac{3}{2 \pi h^{2}}\right)^{\frac{3 n}{2}} \int \mathrm{d} \boldsymbol{x}_{1} \ldots \int \mathrm{d} \boldsymbol{x}_{n-1} \exp \left(-\frac{3}{2 h^{2}} \sum_{j=1}^{n}\left|\boldsymbol{x}_{j}-\boldsymbol{x}_{j-1}\right|^{2}\right)$,

where each integral is taken over $\mathbb{R}^{3}, \boldsymbol{x}_{0}=\boldsymbol{x}^{\prime}$ and $\boldsymbol{x}_{n}=\boldsymbol{x}$. We calculate the integrals in (2.13) iteratively, starting with $\int \mathrm{d} x_{n-1}$ and ending with $\int \mathrm{d} x_{1}$. Since we consider the limit $h \rightarrow 0$, each integral in (2.13) is of Laplace type [4] and the main contribution comes from the straight path

$\Upsilon^{\star}=\left\{\boldsymbol{x}^{\prime}, \boldsymbol{x}_{1}^{\star}, \ldots \boldsymbol{x}_{n-1}^{\star}, \boldsymbol{x}\right\}, \quad$ where $\quad \boldsymbol{x}_{j}^{\star}=\boldsymbol{x}^{\prime}+\frac{j}{n}\left(\boldsymbol{x}-\boldsymbol{x}^{\prime}\right), j=0,1, \ldots n$.

The result is equivalent with Markoff's solution

$$
g_{n}\left(\boldsymbol{x}, \boldsymbol{x}^{\prime}\right)=\frac{h^{2}}{6 \sigma}\left(\frac{3}{2 \pi n h^{2}}\right)^{\frac{3}{2}} \exp \left(-\frac{3}{2 n h^{2}}\left|\boldsymbol{x}-\boldsymbol{x}^{\prime}\right|^{2}\right)
$$

and the Green function follows from (2.9):

$G\left(\boldsymbol{x}, \boldsymbol{x}^{\prime}\right)=\lim _{h \rightarrow 0} \frac{h^{2}}{6 \sigma} \sum_{n=0}^{\infty}\left(\frac{3}{2 \pi n h^{2}}\right)^{\frac{3}{2}} \exp \left(-\frac{3}{2 n h^{2}}\left|\boldsymbol{x}-\boldsymbol{x}^{\prime}\right|^{2}\right)=\frac{1}{4 \pi \sigma\left|\boldsymbol{x}-\boldsymbol{x}^{\prime}\right|}$.

Thus, we obtained the well known formula of the Green function of the Laplace equation in $\mathbb{R}^{3}$. 


\subsection{Medium with one plane interface of discontinuity of the conductivity}

Suppose that we have a medium that has two infinite layers as shown in figure 2:

$$
\sigma(x)=\sigma(z)= \begin{cases}\sigma^{+} & \text {for } z>\xi \\ \sigma^{-} & \text {for } z<\xi\end{cases}
$$

We calculate the Green function corresponding to this medium with the path-integral method described in sections 2.1 and 2.2. Let us define

$$
\Omega^{+}=\{(x, y, z) \mid z>\xi\} \quad \text { and } \quad \Omega^{-}=\{(x, y, z) \mid z<\xi\}
$$

and suppose that $x$ and $x^{\prime} \in \Omega^{+}$. From the result (2.14) we deduce that there are two classical paths that connect $\boldsymbol{x}^{\prime}$ and $\boldsymbol{x}$ in $n$ steps. There is the direct path that has the probability $g_{n}^{(d)}\left(\boldsymbol{x}, \boldsymbol{x}^{\prime}\right)$ given by (2.15), where $\sigma=\sigma^{+}$. The second path reaches $\boldsymbol{x}$ after a reflection at the interface and it has the probability

$$
\begin{aligned}
g_{n}^{(r)}\left(\boldsymbol{x}, \boldsymbol{x}^{\prime}\right)= & D \frac{h^{2}}{6 \sigma^{+}} \frac{\left(z+z^{\prime}-2 \xi\right)}{n} \\
& \times \sum_{p=0}^{n} \int_{-\infty}^{\infty} \int_{-\infty}^{\infty} \mathrm{d} \alpha \mathrm{d} \beta\left(\frac{3}{2 \pi p h^{2}}\right)^{\frac{3}{2}} \mathrm{e}^{-\frac{3}{2 p h^{2}}\left[\left(\alpha-x^{\prime}\right)^{2}+\left(\beta-y^{\prime}\right)^{2}+\left(\xi-z^{\prime}\right)^{2}\right]} \\
& \times\left[\frac{3}{2 \pi(n-p) h^{2}}\right]^{\frac{3}{2}} \mathrm{e}^{-\frac{3}{2(n-p) h^{2}}\left[(x-\alpha)^{2}+(y-\beta)^{2}+(z-\xi)^{2}\right]} .
\end{aligned}
$$

The interpretation of formula (2.18) is as follows. The paths from $x^{\prime}$ to the interface $\mathcal{I}$ are straight, as given by (2.14). One such path reaches the interface at location $(\alpha, \beta, \xi)$ after $p$ steps. We must consider all possible paths so we integrate over $\alpha$ and $\beta$. Next, from the interface, the destination is reached along a straight path that connects $(\alpha, \beta, \xi)$ to $x$ in $n-p$ steps. We consider all possible values of $p$ and take the average as indicated by the sum in (2.18) which is multiplied by the normal velocity $\frac{\left(z+z^{\prime}-2 \xi\right)}{n}$ along all the paths. Finally, $D$ is the coefficient of reflection given in (2.6). Thus, the probability of the reflected path is (see appendix A)

$$
g_{n}^{(r)}\left(\boldsymbol{x}, \boldsymbol{x}^{\prime}\right)=D \frac{h^{2}}{6 \sigma^{+}}\left(\frac{3}{2 \pi n h^{2}}\right)^{\frac{3}{2}} \mathrm{e}^{-\frac{3}{2 n h^{2}}\left[\left(x-x^{\prime}\right)^{2}+\left(y-y^{\prime}\right)^{2}+\left(2 \xi-z-z^{\prime}\right)^{2}\right]} .
$$

Furthermore, the path that has the largest contribution in the evaluation of $g_{n}^{(r)}\left(\boldsymbol{x}, \boldsymbol{x}^{\prime}\right)$ satisfies the Snell law of reflection that says that the angle of incidence equals the angle of reflection. The calculation of the Green function is completed by using equation (2.9):

$G\left(\boldsymbol{x}, \boldsymbol{x}^{\prime}\right)=\frac{1}{4 \pi \sigma^{+}\left|\boldsymbol{x}-\boldsymbol{x}^{\prime}\right|}+D \frac{1}{4 \pi \sigma^{+} \sqrt{\left(x-x^{\prime}\right)^{2}+\left(y-y^{\prime}\right)^{2}+\left(2 \xi-z-z^{\prime}\right)^{2}}}$,

for $\boldsymbol{x}$ and $\boldsymbol{x}^{\prime} \in \Omega^{+}$.

Next, suppose that $\boldsymbol{x}^{\prime} \in \Omega^{+}$and $\boldsymbol{x} \in \Omega^{-}$. All paths that connect $\boldsymbol{x}$ and $\boldsymbol{x}^{\prime}$ cross the interface $\mathcal{I}$, so we obtain

$$
\begin{aligned}
g_{n}^{(t)}\left(\boldsymbol{x}, \boldsymbol{x}^{\prime}\right)= & T \frac{h^{2}}{6 \sigma^{-}} \frac{\left(z^{\prime}-z\right)}{n} \sum_{p=0}^{n} \int_{-\infty}^{\infty} \int_{-\infty}^{\infty} \mathrm{d} \alpha \mathrm{d} \beta\left(\frac{3}{2 \pi p h^{2}}\right)^{\frac{3}{2}} \mathrm{e}^{-\frac{3}{2 p h^{2}}\left[\left(\alpha-x^{\prime}\right)^{2}+\left(\beta-y^{\prime}\right)^{2}+\left(\xi-z^{\prime}\right)^{2}\right]} \\
& \times\left[\frac{3}{2 \pi(n-p) h^{2}}\right]^{\frac{3}{2}} \mathrm{e}^{-\frac{3}{2(n-p) h^{2}}\left[(x-\alpha)^{2}+(y-\beta)^{2}+(\xi-z)^{2}\right]}
\end{aligned}
$$



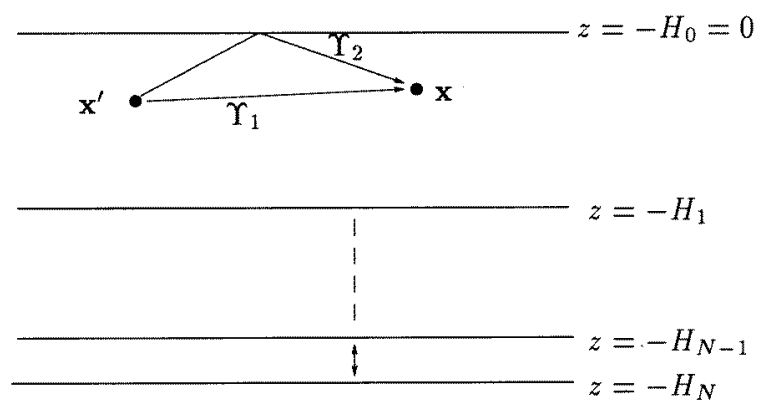

Figure 3. Path diagram for the construction of the term $G_{0}\left(\boldsymbol{x}, \boldsymbol{x}^{\prime}\right)$ in (2.25).

where $T=1-D$ is the coefficient of transmission. The interpretation of (2.21) is similar to the interpretation of (2.18). The result of the calculations in (2.21) give the probability of the transmitted path

$$
g_{n}^{(t)}\left(\boldsymbol{x}, \boldsymbol{x}^{\prime}\right)=T \frac{h^{2}}{6 \sigma^{-}}\left(\frac{3}{2 \pi n h^{2}}\right)^{\frac{3}{2}} \mathrm{e}^{-\frac{3}{2 n h^{2}}\left[\left(x-x^{\prime}\right)^{2}+\left(y-y^{\prime}\right)^{2}+\left(z-z^{\prime}\right)^{2}\right]},
$$

where the main contribution comes from the straight path that connects $x$ to $x^{\prime}$ through the interface. The Green function is given by

$$
G\left(x, x^{\prime}\right)=T \frac{1}{4 \pi \sigma^{-}\left|x-x^{\prime}\right|} \quad \text { for } \quad x \in \Omega^{-} \quad \text { and } \quad x^{\prime} \in \Omega^{+} .
$$

\subsection{Multiscattering series expansion of the Green function in a layered medium occupying} the half-space $\Omega$

The Green function associated with problem (1.1) satisfies

$$
\begin{aligned}
& \nabla \cdot\left[\sigma(z) \nabla G\left(\boldsymbol{x}, \boldsymbol{x}^{\prime}\right)\right]=-\delta\left(\boldsymbol{x}-\boldsymbol{x}^{\prime}\right), \quad \text { for } \quad \boldsymbol{x}, \boldsymbol{x}^{\prime} \in \Omega \\
& G\left(\boldsymbol{x}, \boldsymbol{x}^{\prime}\right) \rightarrow 0 \quad \text { as } \quad\left|\boldsymbol{x}-\boldsymbol{x}^{\prime}\right| \rightarrow \infty . \\
& \frac{\partial G}{\partial z}=0 \quad \text { for } \quad z=0 \quad \text { and } \quad \frac{\partial G}{\partial z^{\prime}}=0 \quad \text { for } \quad z^{\prime}=0 .
\end{aligned}
$$

Furthermore, $G, \sigma \frac{\partial G}{\partial z}$ and $\sigma \frac{\partial G}{\partial z^{\prime}}$ are continuous at any interface between two layers. The conductivity $\sigma(z)$ for $z \leqslant 0$ is given by (1.6) and it is extended to $\sigma=0$ for $z>0$. Thus, the top surface acts as a perfect reflector with reflection coefficient $D_{0}=\frac{\sigma_{1}-0}{\sigma_{1}+0}=1$. Suppose that both $\boldsymbol{x}$ and $\boldsymbol{x}^{\prime}$ belong to the first layer of conductivity $\sigma_{1}$. We use the results of sections 2.1-2.3 and write that the Green function is given by the multiscattering series

$$
G\left(\boldsymbol{x}, \boldsymbol{x}^{\prime}\right)=\sum_{r=0}^{\infty} G_{r}\left(\boldsymbol{x}, \boldsymbol{x}^{\prime}\right),
$$

where $G_{r}$ denotes the term in the series that accounts for a total of $r$ reflections at any of the inner layers $z=-H_{j}, j=1, \ldots, N$. We explain the construction of each term in the series through path diagrams.

Take $r=0$ and observe from the diagram in figure 3 that there are two classical paths that connect $\boldsymbol{x}^{\prime}$ and $\boldsymbol{x}$. The first path $\left(\Upsilon_{1}\right)$ is direct and the other $\left(\Upsilon_{2}\right)$ is reflected at the top surface. Thus, according to the results in section 2.3 and the fact that the reflected path $\Upsilon_{2}$ has amplitude $D_{0}=1$ we have

$$
G_{0}=\frac{1}{4 \pi \sigma_{1} \sqrt{\left(x-x^{\prime}\right)^{2}+\left(y-y^{\prime}\right)^{2}+\left(z-z^{\prime}\right)^{2}}}+\frac{1}{4 \pi \sigma_{1} \sqrt{\left(x-x^{\prime}\right)^{2}+\left(y-y^{\prime}\right)^{2}+\left(z+z^{\prime}\right)^{2}}} .
$$



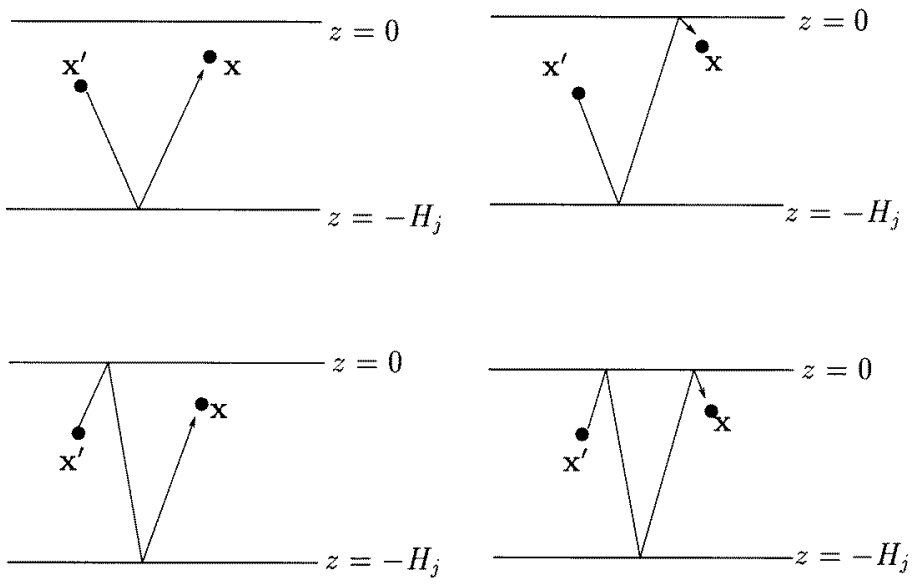

Figure 4. Path diagram for the construction of the term $G_{1}\left(\boldsymbol{x}, \boldsymbol{x}^{\prime}\right)$ in (2.25).

Next, take $r=1$ or, equivalently, consider the contribution to $G\left(\boldsymbol{x}, \boldsymbol{x}^{\prime}\right)$ of all paths that reflect once in the interior of $\Omega$. We must consider the four possibilities shown in the diagrams in figure 4 . As we had before, the reflection at the top surface means multiplication by $D_{0}=1$. Furthermore, the amplitude of a path that reflects at the interface $z=-H_{j}$ is given by the product between the coefficient of reflection $D_{j}=\frac{\sigma_{j}-\sigma_{j+1}}{\sigma_{j}+\sigma_{j+1}}$ and the coefficients of transmission back and forth through the layers at $z=-H_{i}$, where $1 \leqslant i \leqslant j-1$. Thus, the amplitude of each path in the diagrams of figure 4 is $D_{j} \prod_{i=1}^{j-1}\left(1-D_{j}^{2}\right)$. The term in the series (2.25) that corresponds to $r=1$ is

$$
\begin{aligned}
G_{1}=\sum_{j=1}^{N} D_{j} \prod_{i=1}^{j-1}\left(1-D_{j}^{2}\right)\left[\frac{1}{4 \pi \sigma_{1} \sqrt{\left(x-x^{\prime}\right)^{2}+\left(y-y^{\prime}\right)^{2}+\left(2 H_{j}+z+z^{\prime}\right)^{2}}}\right. \\
+\frac{1}{4 \pi \sigma_{1} \sqrt{\left(x-x^{\prime}\right)^{2}+\left(y-y^{\prime}\right)^{2}+\left(2 H_{j}+z-z^{\prime}\right)^{2}}} \\
+\frac{1}{4 \pi \sigma_{1} \sqrt{\left(x-x^{\prime}\right)^{2}+\left(y-y^{\prime}\right)^{2}+\left(2 H_{j}-z+z^{\prime}\right)^{2}}} \\
\left.+\frac{1}{4 \pi \sigma_{1} \sqrt{\left(x-x^{\prime}\right)^{2}+\left(y-y^{\prime}\right)^{2}+\left(2 H_{j}-z-z^{\prime}\right)^{2}}}\right] .
\end{aligned}
$$

The term $G_{2}$ in (2.25) accounts for the contribution of paths such as $\Upsilon$ shown in figure 5. Note that the other three possibilities due to the perfectly reflecting top surface must be included. Finally, we show the diagrams in figure 6 which illustrate the paths that contribute to the term $G_{3}$ in (2.25). Again, the additional possibilities due to the perfectly reflecting top surface must be included.

The construction can be done, in principle, for any $r$. For each path in the diagram, we calculate its amplitude as the product of the coefficients of reflection and transmission through the interfaces. Note that the amplitude of each path belongs to the interval $[-1,1]$. Furthermore, each term in the expression of $G_{r}\left(\boldsymbol{x}, \boldsymbol{x}^{\prime}\right)$ is proportional to the product of $r$ 


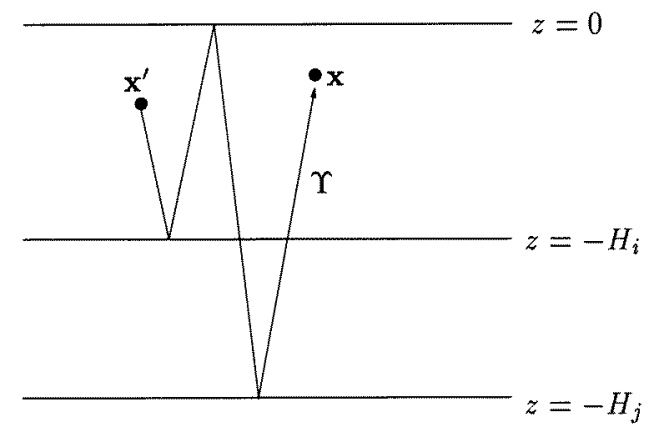

Figure 5. Path diagram for the construction of the term $G_{2}\left(\boldsymbol{x}, \boldsymbol{x}^{\prime}\right)$ in (2.25).

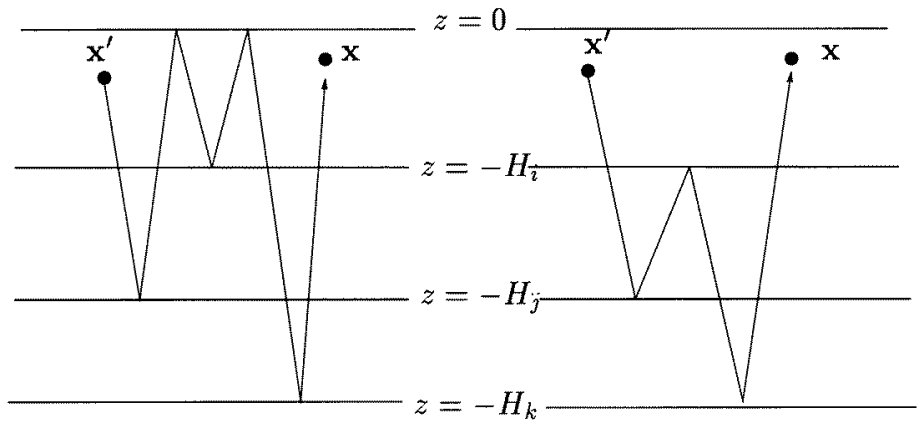

Figure 6. Path diagram for the construction of the term $G_{3}\left(\boldsymbol{x}, \boldsymbol{x}^{\prime}\right)$ in $(2.25)$.

coefficients of reflection $D_{j}$, where

$$
D_{j}=\frac{\sigma_{j}-\sigma_{j+1}}{\sigma_{j}+\sigma_{j+1}} \in[-1,1] .
$$

Thus, if the contrast is high, the reflection coefficients are close to \pm 1 and the convergence of the series is slow. However, for moderate contrast, we expect a fast convergence. Note also that the contribution to the Green function of each path is inversely proportional to the length of the path. Thus, the higher $r$ is, the longer the paths and the smaller the terms in the expression of $G_{r}$. This is of big importance in the convergence of the series as we show in section 3.

\subsection{Discussion}

The multiscattering series expansion (2.25) of the Green function can also be obtained directly from equation (1.7), where

$$
\int_{-\infty}^{\infty} \int_{-\infty}^{\infty} G\left(x-x^{\prime}, y-y^{\prime}, z, z^{\prime}\right) \mathrm{e}^{\mathrm{i} k \cdot\left(x-x^{\prime}, y-y^{\prime}\right)} \mathrm{d} x \mathrm{~d} y=\frac{1}{|\boldsymbol{k}| \sigma_{1}}\left[\frac{1+R(|\boldsymbol{k}|, \boldsymbol{\sigma}, \boldsymbol{d}) \mathrm{e}^{-2|k| d_{1}}}{1-R(|\boldsymbol{k}|, \boldsymbol{\sigma}, \boldsymbol{d}) \mathrm{e}^{-2|k| d_{1}}}\right]
$$

and the kernel $R$ is calculated iteratively as given by (1.8) and (1.9). One can expand the righthand side of equation (2.28) into a power series of coefficients $D_{j}=\frac{\sigma_{j}-\sigma_{j+1}}{\sigma_{j}+\sigma_{j+1}}$ and obtain (2.25). Our approach, based on path integration, gives a physical interpretation of the derivation of (2.25). We show that the problem can be regarded as a random walk in an infinite lattice, where 
the transition probability from one node to another depends on the electrical conductivity of the continuum. Furthermore, of all possible paths in such a random walk, we distinguish the preferred or classical paths that give the largest contribution to the Green function. The classical paths, like rays in geometrical optics, reflect at interfaces according to the Snell law of reflection (see equation (2.19)). However, the transmitted paths remain straight and so they do not satisfy Snell's law of refraction. Furthermore, after each reflection and transmission through a layer, the amplitude of the paths decreases as given by the reflection and transmission coefficients defined in (2.6). Thus, (2.25) is very similar to the Bremmer series obtained by Bremmer $[7,8]$ and by Keller and Keller [22] in studies of wave propagation in layered media. However, there are some differences between the Bremmer series and (2.25). First, the reflection and transmission coefficients do not depend on the angle of incidence and, as explained above, the transmitted paths remain straight while passing through an interface between two layers of different conductivity. The second difference is that each term in (2.25) is exponentially decaying with the length of the path. There is no concept of time of arrival at the surface (as for waves) and all the paths contribute simultaneously to the response at the boundary.

Finally, we mention that equations (2.9) and (2.10) hold for any function $\sigma(x)$ and so, the path integration approach introduced in this section might be useful in deriving approximations of the Green function in media that are more general than the layered ones. In future research, we concentrate on extending the ideas in this section to such media. One example, encountered in mine detection applications, is a medium with a few conducting or insulating inclusions in a uniform background.

\section{Inversion algorithm}

\subsection{Formulation of the nonlinear output least-squares problem}

Given the Green function for problem (1.1) in a layered medium with conductivity given by (1.6), the electric potential on the top surface $S$ is

$$
v(x, y)=\int_{S} j\left(x^{\prime}, y^{\prime}\right) G\left(x^{\prime}, y^{\prime}, z^{\prime}=0 ; x, y, z=0\right) \mathrm{d} x^{\prime} \mathrm{d} y^{\prime} .
$$

The Green function is given by the multiscattering series (2.25). The calculation of $v(x, y)$ by formula (3.1) requires evaluations of integrals like

$$
I(x, y, \xi)=\int_{S} j\left(x^{\prime}, y^{\prime}\right) \frac{2}{4 \pi \sigma_{1} \sqrt{\left(x-x^{\prime}\right)^{2}+\left(y-y^{\prime}\right)^{2}+\xi^{2}}} \mathrm{~d} x^{\prime} \mathrm{d} y^{\prime},
$$

that are computed as follows. The integral in (3.2) is given by

$$
I(x, y, \xi)=\psi(x, y, \xi)
$$

where $\psi$ is the solution of the conductivity problem in a homogeneous medium with $\sigma=\sigma_{1}$ :

$$
\begin{aligned}
& \Delta \psi(x, y, z)=0 \quad \text { in } \Omega \\
& \sigma_{1} \frac{\partial \psi}{\partial z}(x, y, 0)=j(x, y) \quad \text { on } S \\
& \psi, \quad \frac{\partial \psi}{\partial x} \text { and } \frac{\partial \psi}{\partial y} \rightarrow 0 \quad \text { for } \quad x, y \rightarrow \pm \infty \text { or } z \rightarrow-\infty .
\end{aligned}
$$

The calculations are simpler in the Fourier space, where

$$
\hat{\psi}(\boldsymbol{k}, z)= \begin{cases}\frac{J(\boldsymbol{k})}{|\boldsymbol{k}| \sigma_{1}} \mathrm{e}^{|\boldsymbol{k}| z} & \text { for } z \leqslant 0 \text { and } \boldsymbol{k} \neq \mathbf{0} \\ 0 & \text { otherwise. }\end{cases}
$$


Thus, the Fourier coefficients of the measured voltage on the top surface, for $\boldsymbol{k} \neq \mathbf{0}$, are given by (2.25) and (3.1) as

$$
\begin{aligned}
\hat{v}(\boldsymbol{k})=\frac{J(\boldsymbol{k})}{|\boldsymbol{k}| \sigma_{1}}\{ & 1+2 \sum_{i=1}^{N} D_{i} \prod_{j=1}^{i-1}\left(1-D_{j}^{2}\right) \mathrm{e}^{-2|\boldsymbol{k}| H_{i}} \\
& \left.+2 \sum_{i=1}^{N} D_{i} \prod_{j=1}^{i-1}\left(1-D_{j}^{2}\right) \sum_{k=1}^{N} D_{k} \prod_{l=1}^{k-1}\left(1-D_{l}^{2}\right) \mathrm{e}^{-2|\boldsymbol{k}|\left(H_{i}+H_{k}\right)}+\mathrm{O}\left(D^{3}\right)\right\},
\end{aligned}
$$

where by $\mathrm{O}\left(D^{3}\right)$ we mean the contribution of the terms $G_{r}, r \geqslant 3$ in the multiscattering series (2.25). The series expansion (3.6) of the voltage $\hat{v}(\boldsymbol{k})$ is convergent because of the decaying exponentials. Indeed, the higher the terms in the series, the longer the associated paths and the smaller the exponentials. Furthermore, all the exponentials are multiplied by the amplitude associated with each path and each amplitude has a magnitude of at most one. For moderate contrasts, the reflection coefficients are small and we expect that it is sufficient to consider only the first few terms in (3.6).

In our numerical experiments, the domain $\mathcal{M} \subset S$ of measurements is a square of length $L$, over which we place electrodes on a uniform mesh with $N_{E}$ nodes on each side. The number of frequencies that give independent equations such as (3.6) are $M=\frac{1}{2}\left(\frac{N_{E}}{2}+1\right)\left(\frac{N_{E}}{2}+2\right)-1$. Thus, our inversion algorithm attempts to find the reflection coefficients $D_{i}, i=1, \ldots N$ that satisfy (3.6) in the least-squares sense, for the frequencies

$$
\boldsymbol{k}=\left(\frac{2 \pi}{L} i, \frac{2 \pi}{L} j\right), \quad \text { for } \quad i=0, \ldots, \frac{N_{E}}{2} ; \quad j=i, \ldots, \frac{N_{E}}{2} \quad \text { and } \quad \boldsymbol{k} \neq \mathbf{0} .
$$

In general, is is easy to get the conductivity on the surface, so we assume that we know $\sigma_{1}$. The inversion algorithm proceeds to reconstruct the reflection coefficients step by step. The first step takes into account only single reflections in the interior of $\Omega$ and terms in (3.6) of order $D$. Thus, the first step of our inversion algorithm implies solving the linear least-squares problem

$$
\min _{D}\|B D-b\|_{2}
$$

where

$$
\begin{aligned}
& \boldsymbol{D}=\left(D_{1}, \ldots, D_{N}\right)^{T} \in \mathbb{R}^{N} \\
& B_{i j}=2 \exp \left(-2\left|\boldsymbol{k}_{i}\right| H_{j}\right), \quad \text { for } \quad i=1, \ldots M \text { and } j=1, \ldots N \\
& b_{i}=\left|\boldsymbol{k}_{i}\right| \sigma_{1} \operatorname{real}\left(\frac{\hat{v}\left(\boldsymbol{k}_{i}\right)}{J\left(\boldsymbol{k}_{i}\right)}\right)-1, \quad \text { for } \quad i=1, \ldots M .
\end{aligned}
$$

We solve (3.9) with the singular value decomposition [17]. In general, $B$ has singular values that decay quickly to zero. To decide how many singular values to use in the leastsquares solution, we use that, in general, the measurements of the potential $v$ are noisy. Let us denote by $\tilde{v}(\boldsymbol{k})$ the noisy Forier coefficients, where

$$
\tilde{v}\left(\boldsymbol{k}_{i}\right)=\hat{v}\left(\boldsymbol{k}_{i}\right)\left(1+\varepsilon_{i}\right), \quad i=1, \ldots M, \quad\left|\varepsilon_{i}\right| \leqslant \varepsilon,
$$

and $\varepsilon$ is the noise level. Then, the vector $\boldsymbol{b}$ in (3.8) is perturbed to

$\tilde{\boldsymbol{b}}=\boldsymbol{b}+\Delta \boldsymbol{b}, \quad$ where $\quad\left|\Delta b_{i}\right| \approx\left|\boldsymbol{k}_{i}\right| \sigma_{1}\left|\frac{\tilde{v}\left(\boldsymbol{k}_{i}\right)}{J\left(\boldsymbol{k}_{i}\right)}\right|\left|\varepsilon_{i}\right| \leqslant\left|1+\tilde{b}_{i}\right| \varepsilon, \quad i=1, \ldots M$.

Thus,

$$
\|B D-\tilde{b}\|_{2} \lesssim\|B D-b\|_{2}+\varepsilon\|1+\boldsymbol{b}\|_{2}
$$


and we accept $\boldsymbol{D}$ as the least-squares solution of (3.9) if

$$
\|B \boldsymbol{D}-\tilde{\boldsymbol{b}}\|_{2} \approx \varepsilon\|1+\boldsymbol{b}\|_{2} .
$$

The solution $\boldsymbol{D}$ is a first approximation of the reflection coefficients. Once we have $\boldsymbol{D}$, we find an approximation of the electrical conductivity as

$$
\sigma_{i+1}=\sigma_{i}\left(\frac{1-D_{i}}{1+D_{i}}\right), \quad i=1, \ldots N
$$

The next step of the inversion, takes in (3.6) terms of order up to $D^{2}$. This leads to the nonlinear least-squares problem

$$
\begin{aligned}
\min _{\boldsymbol{D}} \sum_{i=1}^{M} r_{i}^{2}(\boldsymbol{D}), & \text { where } \\
r_{i}(\boldsymbol{D}) & =b_{i}-2 \sum_{j=1}^{N} D_{j} \mathrm{e}^{-2\left|k_{i}\right| H_{j}}-2 \sum_{j=1}^{N} \sum_{k=1}^{N} D_{j} D_{k} \mathrm{e}^{-2\left|k_{i}\right|\left(H_{j}+H_{k}\right)} .
\end{aligned}
$$

We solve (3.13) with Newton's method, where the initial guess is the solution of (3.9). Next, we take in (3.6) terms of order $D^{3}$ that account for at most three reflections inside $\Omega$. The least-squares problem to solve is:

$$
\begin{aligned}
& \min _{\boldsymbol{D}} \sum_{i=1}^{M} r_{i}^{2}(\boldsymbol{D}), \quad \text { where } \\
& r_{i}(\boldsymbol{D})=b_{i}-2 \sum_{j=1}^{N} D_{j}\left(1-\sum_{k=1}^{j-1} D_{k}^{2}\right) \mathrm{e}^{-2\left|\boldsymbol{k}_{i}\right| H_{j}} \\
& -2 \sum_{j=1}^{N} \sum_{k=1}^{N} D_{j} D_{k} \mathrm{e}^{-2\left|k_{i}\right|\left(H_{j}+H_{k}\right)}-2 \sum_{j=1}^{N} \sum_{k=1}^{N} \sum_{p=1}^{N} D_{j} D_{k} D_{p} \mathrm{e}^{-2\left|k_{i}\right|\left(H_{j}+H_{k}+H_{p}\right)} \\
& +2 \sum_{j=1}^{N} \sum_{k=1}^{j-1} \sum_{p=k+1}^{N} D_{j} D_{k} D_{p} \mathrm{e}^{-2\left|k_{i}\right|\left(H_{j}-H_{k}+H_{p}\right)} .
\end{aligned}
$$

This can be done for higher and higher terms in the series (3.6). For each step, we have a nonlinear least-squares problem that we solve with Newton's method, where the initial guess is the solution of the previous step. If the vector $\boldsymbol{D}$ does not change much from one step to another, we accept it as the solution of the inverse problem and calculate the conductivity distribution from (3.12).

In summary, the inversion algorithm introduced in this section consists of a sequence of least-squares problems. The first problem (3.8) is linear and all the others, like (3.13) and (3.14), are nonlinear. In each least-squares problem, we consider an additional term $G_{r}$ in the series expansion (2.25) of the Green function. As shown by (2.25) and (3.6), each term $G_{r}$ has a strength proportional to the $r$ th power of the reflection coefficients $D_{j}$. For a finite contrast in $\sigma,\left|D_{j}\right|<1$ for all $j=1, \ldots N$ and so $\left|G_{r}\right|$ is expected to be smaller than $\left|G_{r-1}\right|$. Furthermore, $G_{r}$ contains longer paths than $G_{r-1}$, and, due to the exponentially decaying factors in (3.6), is less important than $G_{r-1}$ in the approximation of the Green function. Thus, for an intermediate contrast, the solution of the least-squares problem that uses $G\left(\boldsymbol{x}, \boldsymbol{x}^{\prime}\right) \approx \sum_{i=0}^{r-1} G_{i}\left(\boldsymbol{x}, \boldsymbol{x}^{\prime}\right)$ is expected to be a reasonable approximation of the conductivity function $\sigma(\boldsymbol{x})$ and so a good initial guess for the next nonlinear least-squares problem that uses the additional term $G_{r}\left(\boldsymbol{x}, \boldsymbol{x}^{\prime}\right)$ in the approximation of the Green function. Having a good initial guess, each nonlinear leastsquares problem is solved easily, in just a few iterations. 


$$
z=-H_{0}=0
$$

$\sigma_{1}$

$$
z=-H_{1}
$$

$$
\sigma_{2}=\sigma_{1}+\gamma
$$

Figure 7. Medium with two layers of conductivity $\sigma_{1}$ and $\sigma_{2}$, respectively.

\subsection{Comparison with the Born series}

In this section we compare the multiscattering series (3.6) with the popular Born series that is based on the linearization of the equations about a reference conductivity. For simplicity, we make the comparison for a medium with two layers, as shown in figure 7:

$$
\sigma(z)= \begin{cases}\sigma_{1} & \text { for }-H_{1}<z \leqslant 0 \\ \sigma_{2}=\sigma_{1}+\gamma & \text { for } z \leqslant-H_{1}\end{cases}
$$

The exact expression of the Fourier coefficients of the voltage on the top surface is

$$
\hat{v}(\boldsymbol{k})=\frac{J(\boldsymbol{k})}{|\boldsymbol{k}| \sigma_{1}}\left[\frac{1+D_{1} \mathrm{e}^{-2|\boldsymbol{k}| H_{1}}}{1-D_{1} \mathrm{e}^{-2|\boldsymbol{k}| H_{1}}}\right], \quad \boldsymbol{k} \neq \mathbf{0},
$$

where

$D_{1}=-\frac{\gamma}{2 \sigma_{1}+\gamma} \quad$ and $\quad\left|D_{1}\right| \mathrm{e}^{-2|k| H_{1}}<1 \quad$ for any $\gamma \quad$ and $\quad H_{1}>0$

Thus, we write the series expansion of $\left(1-D_{1} \mathrm{e}^{-2|k| H_{1}}\right)^{-1}$ in (3.16) and obtain

$\hat{v}(\boldsymbol{k})=\frac{J(\boldsymbol{k})}{|\boldsymbol{k}| \sigma_{1}}\left[1+2 D_{1} \mathrm{e}^{-2|\boldsymbol{k}| H_{1}}+2 D_{1}^{2} \mathrm{e}^{-4|\boldsymbol{k}| H_{1}}+2 D_{1}^{3} \mathrm{e}^{-6|k| H_{1}}+\mathrm{O}\left(D_{1}^{4}\right)\right]$,

which is exactly the multiscattering series (3.6).

Next, we write the Born series for imaging the conductivity (3.15). In an iterative Born method, the potential $\phi$ is given by [13]

$$
\begin{gathered}
\phi^{(n+1)}(\boldsymbol{x})=\psi(\boldsymbol{x})-\gamma \int_{\Omega_{\gamma}} \nabla_{x^{\prime}} \phi^{(n)}\left(\boldsymbol{x}^{\prime}\right) \cdot \nabla_{x^{\prime}} G_{0}\left(\boldsymbol{x}, \boldsymbol{x}^{\prime}\right) \mathrm{d} \boldsymbol{x}^{\prime}, \quad \boldsymbol{x} \in \Omega, \quad n=1,2, \ldots \\
\phi^{(0)}(\boldsymbol{x})=\psi(\boldsymbol{x}) \\
\Omega_{\gamma}=\text { support of } \gamma
\end{gathered}
$$

where $\psi$ is the solution of problem (3.4) in a homogeneous medium and $G_{0}$ is the Green function associated with it and given by (2.26). Integration by parts gives the first step approximation of the electric potential as

$\phi^{(1)}(\boldsymbol{x})=\psi(\boldsymbol{x})-\gamma \int_{-\infty}^{\infty} \int_{-\infty}^{\infty} G_{0}\left(x^{\prime}, y^{\prime},-H_{1} ; \boldsymbol{x}\right) \frac{\partial \psi}{\partial z^{\prime}}\left(x^{\prime} \cdot y^{\prime},-H_{1}\right) \mathrm{d} x^{\prime} \mathrm{d} y^{\prime}$

and, after straightforward calculations, we have

$$
\begin{aligned}
& \phi^{(1)}(\boldsymbol{x})=\psi(\boldsymbol{x})-\frac{\gamma}{\sigma_{1}} F(\boldsymbol{x}), \quad \text { where } F \text { is the harmonic function } \\
& F(x, y, z)=\frac{1}{2 \pi} \int_{-\infty}^{\infty} \int_{-\infty}^{\infty} \frac{J(\boldsymbol{k})}{|\boldsymbol{k}| \sigma_{1}} \mathrm{e}^{|\boldsymbol{k}|\left(z-H_{1}\right)} \cosh \left(|\boldsymbol{k}| H_{1}\right) \mathrm{e}^{-\mathrm{i} k \cdot(x, y)} \mathrm{d} \boldsymbol{k} .
\end{aligned}
$$


The calculations of $\phi^{(2)}, \phi^{(3)}$ and so on are similar to the above and the iterative Born method gives the Fourier coefficient of the potential at the surface:

$\hat{\phi}^{(n)}(\boldsymbol{k}, z=0)=\frac{J(\boldsymbol{k})}{|\boldsymbol{k}| \sigma_{1}}\left\{1+\sum_{p=1}^{n}(-1)^{p}\left(\frac{\gamma}{\sigma_{1}}\right)^{p} \mathrm{e}^{-p|\boldsymbol{k}| H_{1}}\left[\cosh \left(|\boldsymbol{k}| H_{1}\right)\right]^{p}\right\}, \quad n=1,2, \ldots$

Thus, the Born series converges only if

$$
\left|\frac{\gamma}{\sigma_{1}}\right| \mathrm{e}^{-|k| H_{1}} \cosh \left(|k| H_{1}\right)<1,
$$

or, equivalently, if the contrast is limited to

$$
\left|\frac{\gamma}{\sigma_{1}}\right|<\frac{2}{1+\mathrm{e}^{-|k| H_{1}}}
$$

whereas the multiscattering series (3.6) converges unconditionally. Furthermore, the first step of our inversion algorithm

$$
\hat{v}(\boldsymbol{k}) \approx \frac{J(\boldsymbol{k})}{|\boldsymbol{k}| \sigma_{1}}\left[1-\frac{\gamma}{2 \sigma_{1}+\gamma} \mathrm{e}^{-2|\boldsymbol{k}| H_{1}}\right]
$$

is clearly different from the first step of the Born algorithm.

\subsection{Discretization, penetration and resolution}

In inversion, both the values of the conductivity in the layers and the location of the layers are unknown. To solve the inverse problem, we discretize the medium by defining a mesh along the $z$ direction, for $z \leqslant 0$. The mesh can be uniform or nonuniform and between two mesh points $z=-H_{j-1}$ and $z=-H_{j}$ we assume that the electric conductivity is a constant $\sigma=\sigma_{j}$. In this section we explain how to choose the discretization of the layered medium, or, equivalently, how to define $H_{j}, j=1, \ldots N$.

The first question to address is the depth penetration of each Fourier mode. Let us consider a medium with one layer that has the electrical conductivity

$$
\sigma= \begin{cases}\sigma_{1} & \text { for }-H<z \leqslant 0 \\ \sigma_{2} & \text { for } z \leqslant-H\end{cases}
$$

The Fourier coefficients of the exact solution at the boundary are

$$
\hat{v}(\boldsymbol{k})= \begin{cases}\frac{J(\boldsymbol{k})}{|\boldsymbol{k}| \sigma_{1}}\left[\frac{1+D_{1} \mathrm{e}^{-2|k| H}}{1-D_{1} \mathrm{e}^{-2|\boldsymbol{k}| H}}\right] & \text { for } \boldsymbol{k} \neq \mathbf{0} \\ 0 & \text { otherwise }\end{cases}
$$

where

$$
D_{1}=\frac{\sigma_{1}-\sigma_{2}}{\sigma_{1}+\sigma_{2}} \in[-1,1] .
$$

We compare the response of the medium with conductivity defined by (3.25) to the response of a homogeneous medium of conductivity $\sigma_{1}$ :

$$
\frac{|\hat{v}(\boldsymbol{k})-\hat{\psi}(\boldsymbol{k}, 0)|}{|\hat{\psi}(\boldsymbol{k}, 0)|}=\frac{2\left|D_{1}\right| \mathrm{e}^{-2|k| H}}{1-D_{1} \mathrm{e}^{-2|k| H}} \leqslant \frac{2 \mathrm{e}^{-2|k| H}}{1-\mathrm{e}^{-2|k| H}} .
$$

The discontinuity at $z=-H$ in the conductivity can be felt at the surface if the ratio in (3.27) is larger than the noise level $\varepsilon$. Otherwise, the Fourier mode with frequency $k$ does not see 
$z=0$

$\sigma_{1}$

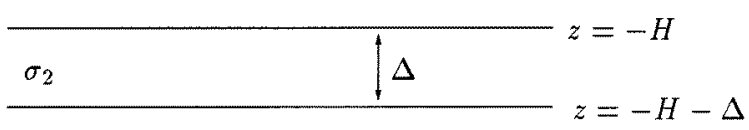

$\sigma_{1}$

Figure 8. Medium with one thin layer of conductivity $\sigma_{2}$ imbedded in a uniform material of conductivity $\sigma_{1}$.

the layer. Based on this result, we define the depth of penetration of the Fourier mode with frequency $\boldsymbol{k}$ as the depth $H(\boldsymbol{k})$ for which the upper bound in (3.27) is equal to the noise level $\varepsilon$ :

$$
H(k)=\frac{1}{2|k|} \ln \left(\frac{2}{\varepsilon}\right) \text {. }
$$

The result (3.22) shows that the high frequency modes explore only the top layers and attenuate quickly with depth. Furthermore, (3.22) gives insight into the question of optimal currents $[13,19,12,15,18]$. From (3.6) it is clear that the residual $w\left(\boldsymbol{k}_{j}\right)=\hat{v}\left(\boldsymbol{k}_{j}\right)-\hat{\psi}\left(\boldsymbol{k}_{j}, 0\right)$ is proportional to the input current $J\left(\boldsymbol{k}_{j}\right)$, where the index $j=1, \ldots M$ denotes any of the relevant frequencies. Thus,

$$
\boldsymbol{W}=\Lambda J, \quad W_{i}=w\left(\boldsymbol{k}_{i}\right), \quad J_{i}=J\left(\boldsymbol{k}_{i}\right), \quad i=1, \ldots M,
$$

where the matrix of responses $\Lambda$ is diagonal:

$$
\Lambda_{i i}=\frac{2}{\sigma_{1}} \sum_{j=1}^{N} \frac{\mathrm{e}^{-2\left|k_{i}\right| H_{j}}}{\left|\boldsymbol{k}_{i}\right|} D_{j}+\mathrm{O}\left(D^{2}\right), \quad i=1, \ldots M .
$$

The reflection coefficients $D_{j}$ are not known so $\Lambda$ cannot be calculated a priori. However, from (3.28) it is clear that to image the conductivity at depths larger than $H$, the current $j$ must have nonzero Fourier coefficients with frequency

$$
|\boldsymbol{k}| \leqslant \frac{1}{2 H} \ln \left(\frac{2}{\varepsilon}\right)
$$

The next question that is relevant to the choice of the discretization of the medium is the resolution. To study resolution, we consider the layered medium shown in figure 8 with electrical conductivity:

$$
\sigma= \begin{cases}\sigma_{1} & \text { for }-H<z \leqslant 0 \text { and } z \leqslant-H-\Delta \\ \sigma_{2} & \text { for }-H-\Delta<z \leqslant-H .\end{cases}
$$

The exact expression of the response voltage at the top surface is

$$
\hat{v}(\boldsymbol{k})=\frac{J(\boldsymbol{k})}{|\boldsymbol{k}| \sigma_{1}}\left\{\frac{1+D \mathrm{e}^{-2|k| H}\left[\frac{1-\mathrm{e}^{-2|k| \Delta}}{\left.1-D^{2} \mathrm{e}^{-2|k| \Delta}\right]}\right.}{1-D \mathrm{e}^{-2|k| H}\left[\frac{1-\mathrm{e}^{-2|k| \Delta}}{1-D^{2} \mathrm{e}^{-2|k| \Delta}}\right]}\right\}, \quad \text { where } \quad D=\frac{\sigma_{1}-\sigma_{2}}{\sigma_{1}+\sigma_{2}} \in[-1,1] .
$$


We compare the response (3.32) of the medium with conductivity (3.31) to the response of a homogeneous medium of conductivity $\sigma_{1}$ :

$$
\begin{aligned}
& \frac{|\hat{v}(\boldsymbol{k})-\hat{\psi}(\boldsymbol{k}, 0)|}{|\hat{\psi}(\boldsymbol{k}, 0)|}=\frac{2|D|}{1-D f} \leqslant \frac{2 f}{1-f}, \\
& \text { where } \quad f(|\boldsymbol{k}|, H, \Delta)=\left[\frac{1-\mathrm{e}^{-2|k| \Delta}}{1-D^{2} \mathrm{e}^{-2|k| \Delta}}\right] \mathrm{e}^{-2|k| H}<1 .
\end{aligned}
$$

The layer can be resolved by the frequency $\boldsymbol{k}$ if the ratio in (3.33) is larger than the noise level $\varepsilon$. We define the resolution limit of the Fourier modes with frequency $k$ as the width $\Delta$ that gives the upper bound in (3.33) equal to the noise level:

$$
\Delta(\boldsymbol{k})=\frac{1}{2|\boldsymbol{k}|} \ln \left(\frac{\mathrm{e}^{-2|k| H}}{\mathrm{e}^{-2|k| H}-\frac{\varepsilon}{2}}\right),
$$

where $H<H(\boldsymbol{k})$ defined by (3.28). Thus, the high frequency modes give a better resolution than the low frequency ones.

Our discretization in $z$ of the domain $\Omega$ takes into consideration the noise level $\varepsilon$, the penetration depths (3.28) and the resolution widths (3.34). For example, suppose that we defined the depths $H_{j}$ for $j=1, \ldots p<N$. We choose the width of the next layer $d_{p+1}=H_{p+1}-H_{p}$ as

$$
d_{p+1}=\max \left(d_{\min }, \Delta\left(\boldsymbol{k}_{\max }\right)\right),
$$

where $\boldsymbol{k}_{\max }$ is the highest available frequency that penetrates to depths larger than $H_{p}$ and $d_{\min }$ is the minimum, user defined, width of the layers. Then, we calculate $H_{p+1}$ and repeat the process until we reach the maximum depth given by (3.28) for the lowest available frequency. This algorithm defines a nonuniform, adaptive discretization that is shown in the next section to give better results than a uniform discretization that does not take into account the resolution and depth of penetration issues.

\section{Numerical results}

In this section we present the numerical results obtained with the inversion algorithm described in section 3. In all the experiments we used synthetic data generated by the formula (1.7) to which we added noise. In [27], the authors discuss the issue of measurement accuracy in real data gathering experiments. The level of noise expected in real data, given the present modern equipment is approximately $1 \%$. Thus, the noise considered in this section is in this range. However, the number $1 \%$ is probably not always accurate and it may be site dependent. In the next section, we test our algorithm for much noisier data. Indeed, in section 5 we consider conductivity functions that are not perfectly layered. This leads to a voltage response on the surface that is different (by as much as $50 \%$ at some points) from that of a perfectly layered medium. The domain $\mathcal{M} \subset S$ of measurements is a square of length $L=20$ and the electrodes are aranged on a uniform mesh with $N_{E}=16$ grid points in each direction. Thus, we have $M=44$ frequencies used in the identification of the conductivity $\sigma(z)$ :

$$
\boldsymbol{k}=\left(\frac{\pi}{10} i, \frac{\pi}{10} j\right), \quad \text { for } \quad i=0, \ldots, 8 ; j=i, \ldots, 8 \text { and } \boldsymbol{k} \neq \mathbf{0} .
$$

In the first experiment we image a medium with two layers and conductivity

$$
\sigma(z)= \begin{cases}1 & \text { for }-0.75<z \leqslant 0 \\ 3 & \text { for } z \leqslant-0.75\end{cases}
$$




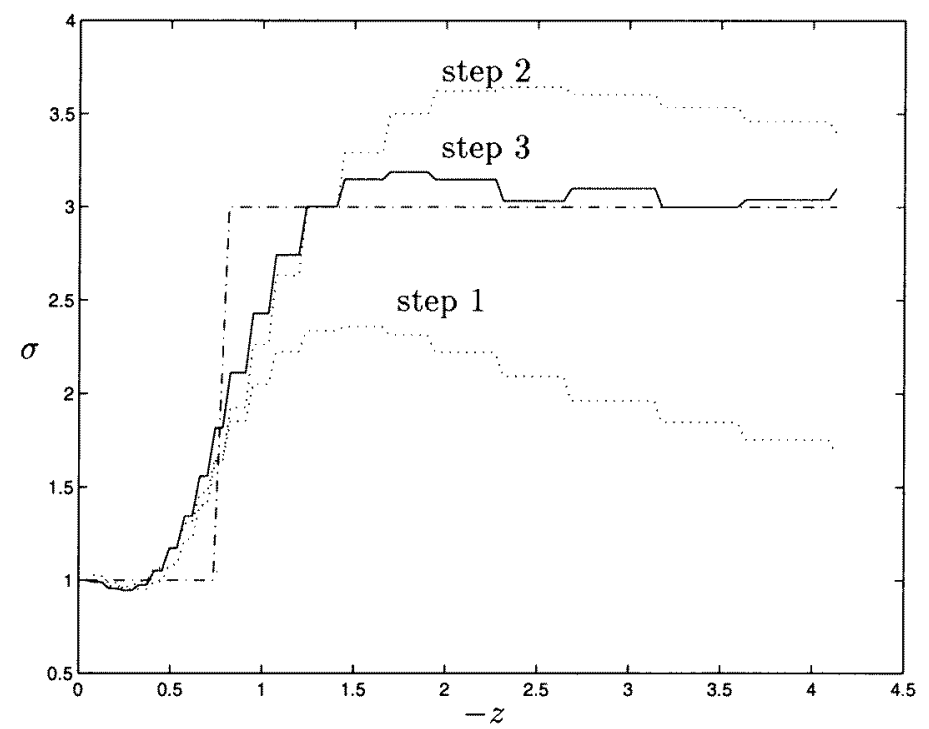

Figure 9. Image of a medium with two layers. Data contain $1 \%$ multiplicative noise. The true conductivity is shown with the dash-dotted line. The conductivity at the final step is shown by the full line and for the intermediate steps we use the dotted lines.

The data contain $1 \%$ multiplicative noise so the maximum penetration is $H_{\max }=8.4325$. In figure 9 we show the image obtained after the first, second and third steps of the inversion algorithm. After the third step, the image remains basically unchanged so the algorithm converged. Thus, for a contrast of $200 \%$ it is sufficient to consider terms of order at most $D^{3}$ (three inner reflections) in the multiscattering series (3.6). Furthermore, from one step of the algorithm to the next, the initial guess for Newton's method in the solution of the nonlinear least-squares problems (3.13) and (3.14) is quite close to the solution. Thus, Newton's method converges in very few steps.

The second experiment tests the effect of the discretization of the medium. We reconstruct the conductivity

$$
\sigma(z)= \begin{cases}1 & \text { for }-0.5<z \leqslant 0 \\ 2 & \text { for } z \leqslant-0.5\end{cases}
$$

from noisy data, with $1 \%$ multiplicative noise. First, we use a uniform mesh in the vertical direction, with spacing $\Delta z=0.12$. The result is shown in figure 10 with the dotted curve. Next, we reconstruct the conductivity with an adaptive mesh defined as in section 3.3, equation (3.35), where the minimum spacing is $d_{\min }=0.12$. The result is shown in figure 10 with the full line. Thus, the image given by the adaptive mesh is superior to the image obtained with the uniform mesh. Near the surface, the uniform mesh spacing is in agreement with the resolution limit defined by (3.34). However, deeper inside the medium, where the high frequency modes cannot penetrate, $\Delta z$ is too small for the large frequencies to resolve and we obtain the oscillations shown in figure 10.

In the third experiment we image a medium with three layers and conductivity

$$
\sigma(z)= \begin{cases}1 & \text { for }-0.5<z \leqslant 0 \\ 4 & \text { for }-1.75<z \leqslant-0.5 \\ 1 & \text { for } z \leqslant-1.75,\end{cases}
$$




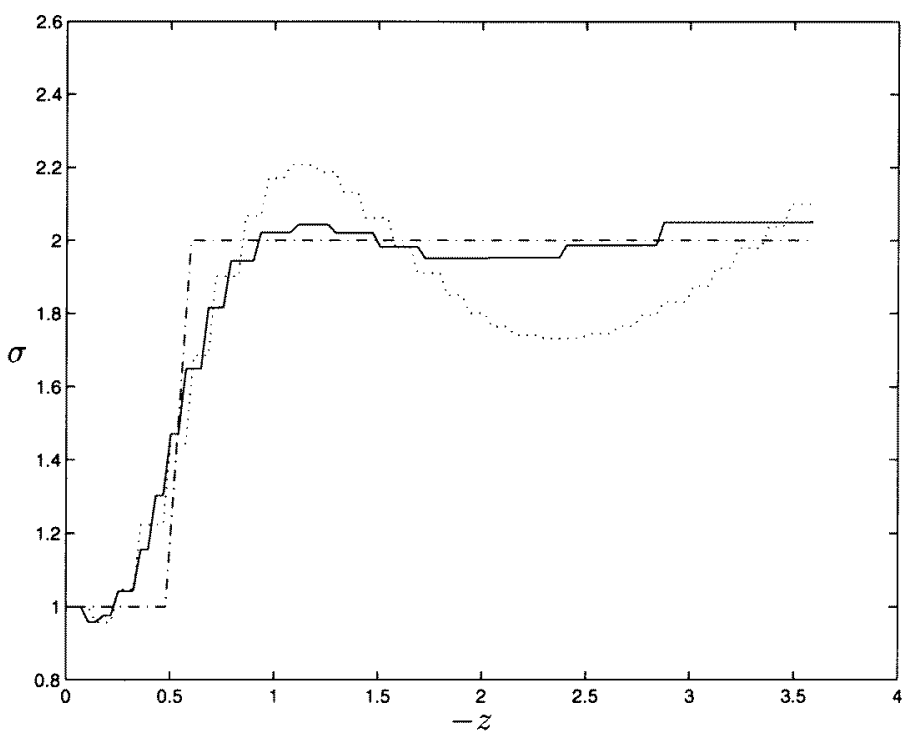

Figure 10. Image of a medium with two layers. Data contain $1 \%$ multiplicative noise. The true conductivity is shown with the dash-dotted line. The conductivity constructed with a uniform mesh is shown with the dotted curve. The conductivity constructed with the adaptive mesh is shown with the full line.

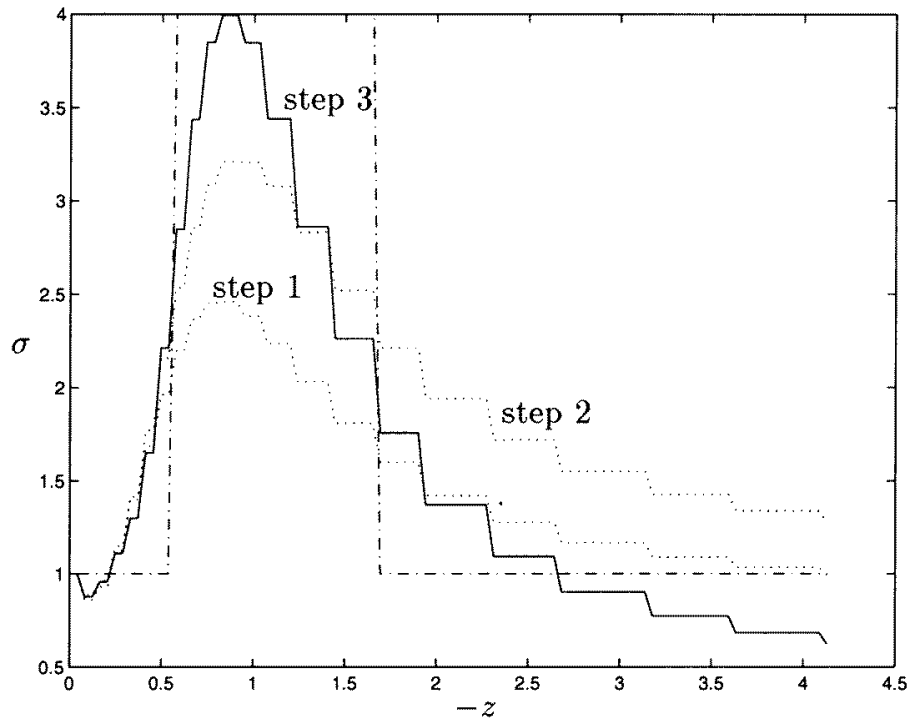

Figure 11. Image of a medium with three layers. Data contain $1 \%$ multiplicative noise. The true conductivity is shown with the dash-dotted line. The conductivity at the final step is shown by the full line and for the intermediate steps we use the dotted lines.

where the data contain $1 \%$ multiplicative noise. The image is shown in figure 11 and the algorithm converged after three steps. The images given after the first and second steps are shown with the dotted lines. We observe that the algorithm correctly locates the inhomogeneity even at the first step. However, the magnitude of $\sigma$ is estimated correctly only after the third step. 


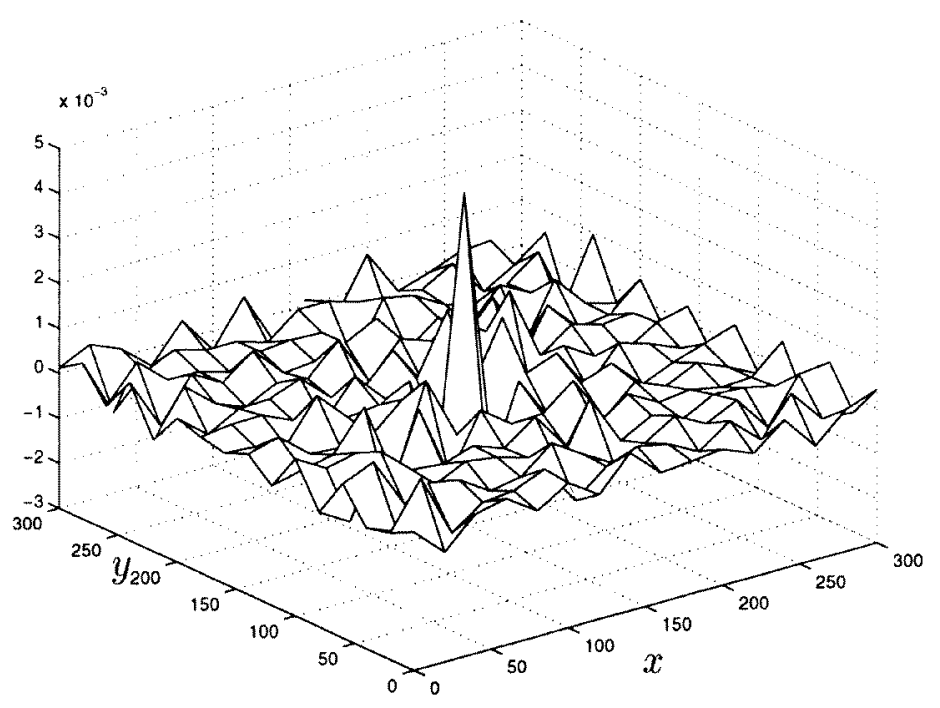

Figure 12. Residual given by the reconstructed conductivity.

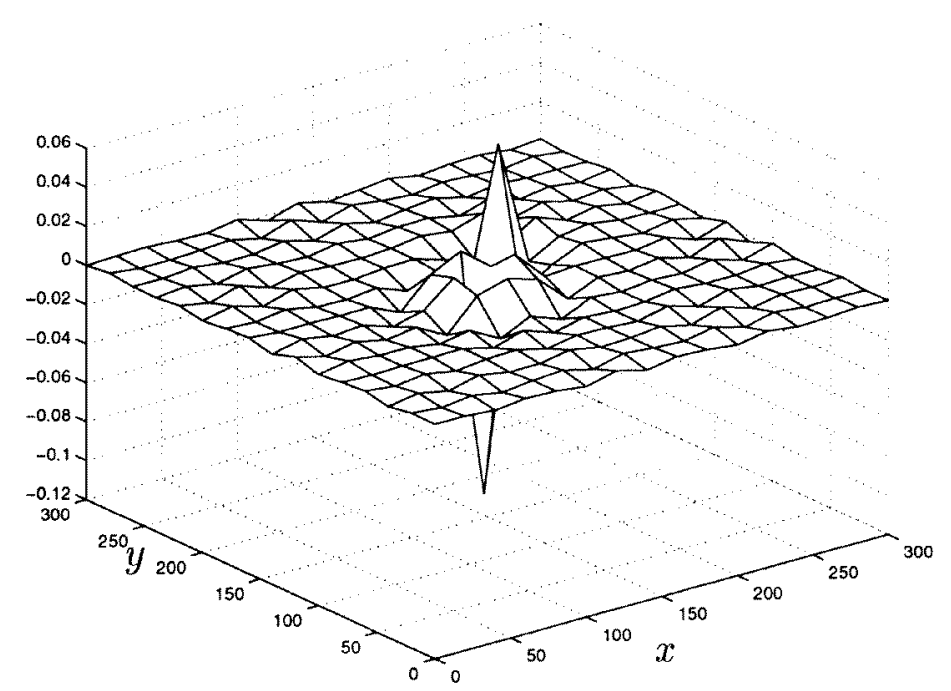

Figure 13. Residual given by the homogeneous medium with conductivity $\sigma=1$.

Next, we use the image obtained to solve for the potential at the top surface and compare it with the data $v(x, y)$. The residual $\phi(x, y, z=0)-v(x, y)$ normalized by $\max _{(x, y) \in \mathcal{M}}|v(x, y)|$ is at the noise level as shown in figure 12. For comparison, we show the initial residual calculated with the potential $\psi(x, y, z=0)$ for a uniform medium with conductivity $\sigma=1$ (figure 13).

In figures 14 and 15 we show the reconstruction of different conductivity distributions of media with five layers. The noise level is $0.5 \%$. Finally, we show in figure 16 the reconstruction of a multilayered, low contrast medium. The data contain $0.5 \%$ noise. The image is quite accurate and the algorithm converges in two steps. In fact, one can see that after the first step of the inversion algorithm, the image (dotted line in figure 16), is very close to the final answer. 


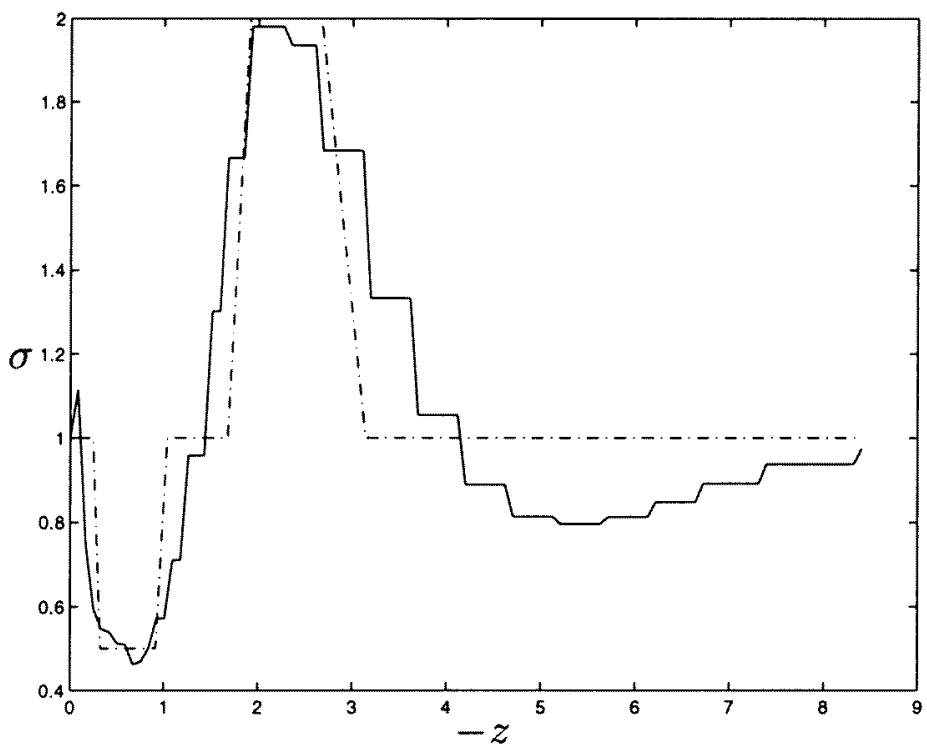

Figure 14. Image of a medium with five layers. Data contain $0.5 \%$ multiplicative noise. The true conductivity is shown with the dash-dotted line. The image obtained is shown with the full line.

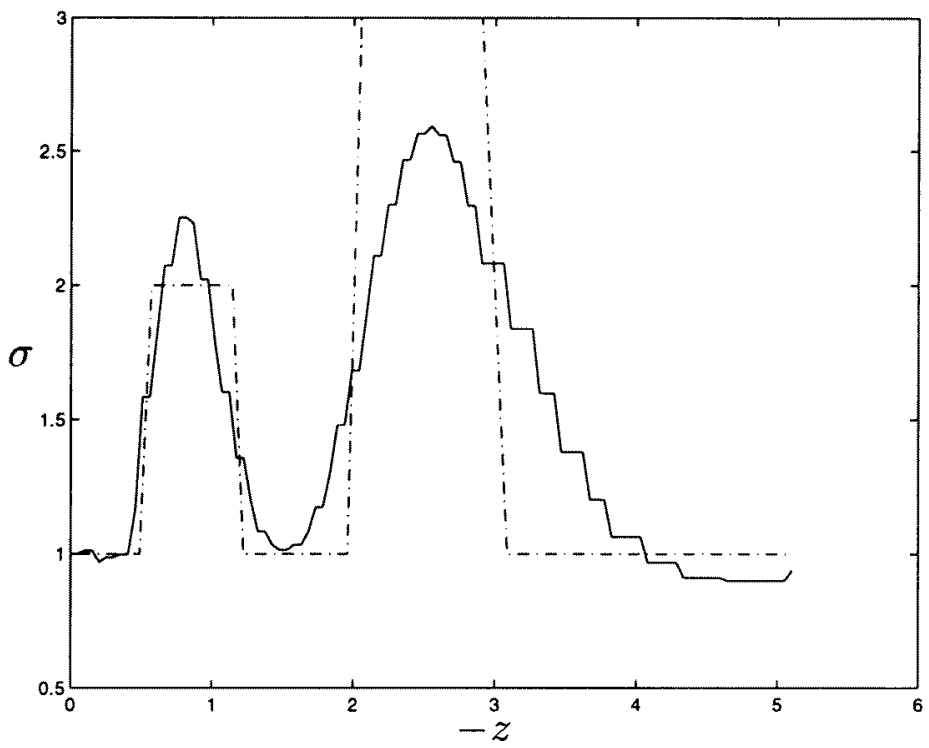

Figure 15. Image of a medium with five layers. Data contain $0.5 \%$ multiplicative noise. The true conductivity is shown with the dash-dotted line. The image obtained is shown with the full line.

In summary, the numerical experiments presented in this section show that our inversion algorithm is successful in recovering conductivities of layered media. We have tested the algorithm in various situations of intermediate contrast and have shown that we can obtain a good quality image in just a few iterations. The algorithm solves at each step a nonlinear least-squares problem and it converges rapidly because we start with a good initial guess that 


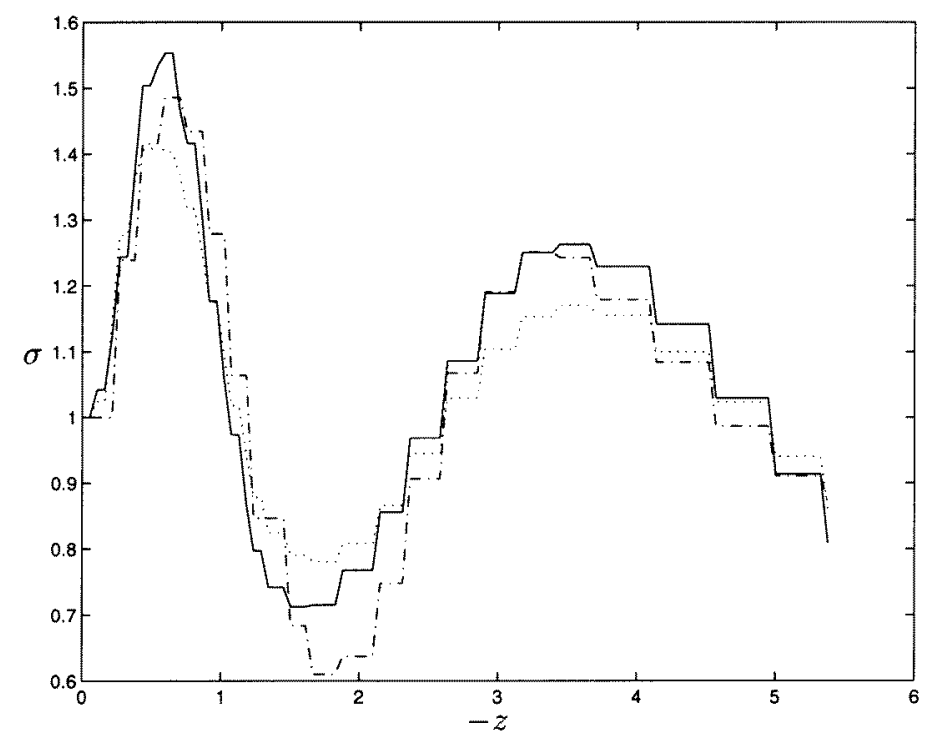

Figure 16. Image of a medium with many layers. Data contain $0.5 \%$ multiplicative noise. The true conductivity is shown with the dash-dotted line. The image is shown with the full line and the conductivity after the first step with the dotted line.

is the solution of the previous iteration. Thus, the performance of our algorithm is similar to that of any nonlinear output least-squares inversion algorithm that starts with a good initial guess $[33,23,24,31]$. However, it is difficult in general to obtain a good initial guess of the conductivity function. Our algorithm addresses this question, by calculating a sequence of initial guesses of $\sigma$, one for each iteration, based on better and better approximations of the Green function. Furthermore, our algorithm is expected to perform better than any inversion algorithm that linearizes the problem, as in a Born approximation [13,3,2]. This is due to the fact that, as shown in section 3.2, the Born series will converge only if the constrast in $\sigma$ is low, typically less than $15 \%$, whereas the series (2.25) is unconditionally convergent. Our algorithm proved to be successful in reconstructing images with contrast as high as $500 \%$. However, as the contrast increases even higher than this, our algorithm will be slower or it could fail. In such situations, we need more and more terms in the series (2.25) to get a good enough approximation of the Green function and so the solution of the first step (3.8) could be very far from the actual $\sigma$. Thus, the following nonlinear least-squares problems might not give any improvements to the image, due to the poor initial guesses given by the previous iterations.

\section{Imaging of almost layered media}

In this section we address the question of sensitivity of our algorithm to the assumption that the medium is perfectly layered. For this purpose, let us consider the conductivity function

$$
\sigma(x)=\alpha(z)+\epsilon \gamma(x), \quad x \in \Omega,
$$

where $\epsilon$ is a small parameter, $\alpha(z)>0$ for any $z \leqslant 0$ and $\gamma(x)$ vanishes on the top surface $\mathrm{S}$. The electric potential $\phi(\boldsymbol{x})$ in a medium with electrical conductivity given by (5.1) has an 
asymptotic expansion [4]

$$
\phi(x)=\sum_{j=0}^{\infty} \epsilon^{j} \phi_{j}(x)=\phi_{0}(x)+\epsilon \psi(x) .
$$

The leading term $\phi_{0}$ in (5.2) satisfies

$$
\begin{aligned}
& \nabla \cdot\left[\alpha(z) \nabla \phi_{0}(x)\right]=0 \quad \text { in } \quad \Omega \\
& \alpha(0) \frac{\partial \phi_{0}}{\partial z}(x, y, 0)=j(x, y), \quad \text { for } \quad(x, y) \in S, \\
& \phi_{0}(x) \rightarrow 0 \quad \text { as } \quad|x| \rightarrow \infty
\end{aligned}
$$

which is identical to equation (1.1). Furthermore, the voltage $\psi$ in (5.2) satisfies

$$
\begin{aligned}
& \nabla \cdot[\alpha(z) \nabla \psi(x)]=-\nabla \cdot\left[\gamma(x) \nabla \phi_{0}(x) \quad \text { in } \Omega\right. \\
& \frac{\partial \psi}{\partial z}(x, y, 0)=0, \quad \text { for } \quad(x, y) \in S \\
& \psi(x) \rightarrow 0 \quad \text { as } \quad|x| \rightarrow \infty .
\end{aligned}
$$

Thus, for small perturbations of the conductivity about the perfectly layered $\alpha(z)$, we use our algorithm to reconstruct $\alpha(z)$. The data are the current $j$ and the voltage $\phi=\phi_{0}+\epsilon \psi$ on the top surface. The voltage $\epsilon \psi$, due to the perturbation $\epsilon \gamma$ in the electrical conductivity, is noise in the imaging process of $\alpha(z)$. After $\alpha(z)$ is approximately found, we can improve the image of $\sigma$ with a typical Born inversion algorithm $[13,3,2]$. This can be done by using, for example, the integral equation formulation [13]

$\psi(\boldsymbol{x})=-\int_{\Omega} \mathrm{d} \boldsymbol{x}^{\prime} \gamma\left(\boldsymbol{x}^{\prime}\right) \int_{\partial \Omega} \mathrm{d} \boldsymbol{y} \nabla_{x^{\prime}} G\left(\boldsymbol{x}, \boldsymbol{x}^{\prime}\right) \cdot \nabla_{x^{\prime}} G\left(\boldsymbol{y}, \boldsymbol{x}^{\prime}\right) \frac{j(\boldsymbol{y})}{\alpha(0)}, \quad \boldsymbol{x} \in S$

that relates the perturbation $\psi$ at the boundary to the unknown $\gamma(x)$ in the domain $\Omega$. The Green function $G\left(\boldsymbol{x}, \boldsymbol{x}^{\prime}\right)$ corresponds to the layered medium with electrical conductivity $\alpha(z)$. Born inversion has been the topic of many studies (see for example [13, 3, 2]). In this section we address only the reconstruction of $\alpha(z)$.

For the purpose of comparison with the results shown in section 4, we take $\alpha(z)$ as shown in figure 10:

$$
\alpha(z)= \begin{cases}1 & \text { for }-0.5 \leqslant z \leqslant 0 \\ 2 & \text { for } z \leqslant-0.5\end{cases}
$$

The perturbation is given by $\gamma(x, y, x)=\cos \left(\frac{2 \pi p x}{L}\right)\left(1-\mathrm{e}^{a z}\right)$, where $L=20$ is the length of the rectangular domain of measurements. We have tested our algorithm for various parameters $a, p$ and $\epsilon$. In figure 17 we show the images obtained for $a=10, p=2, \epsilon=0.1$ and $\epsilon=0.25$, respectively. For a small perturbation, like $\epsilon=0.1$, our algorithm gives a very good image of $\alpha(z)$. For large $\epsilon$, the image deteriorates, as expected. We have also compared the boundary voltage $\phi_{0}(x, y, 0)$ of the perfectly layered medium $\alpha(z)$ to the voltage $\phi(x, y, 0)$ of the perturbed medium $\sigma(x)$. The maximum relative error $\max _{x \in S}\left|\frac{\epsilon \psi(x)}{\phi_{0}(x)}\right|$ is $34.5 \%$ for $\epsilon=0.1$ and $59.2 \%$ for $\epsilon=0.25$, respectively. We repeated the experiment of imaging the layered part of the conductivity, for various perturbations and different models of $\alpha(z)$. All the experiments show that, if the perturbation $\sigma(x)-\alpha(z)$ is small, our algorithm obtains a good quality image of $\alpha(z)$. This is also expected to hold for media with layers that are not perfectly horizontal, but have a small dip. 


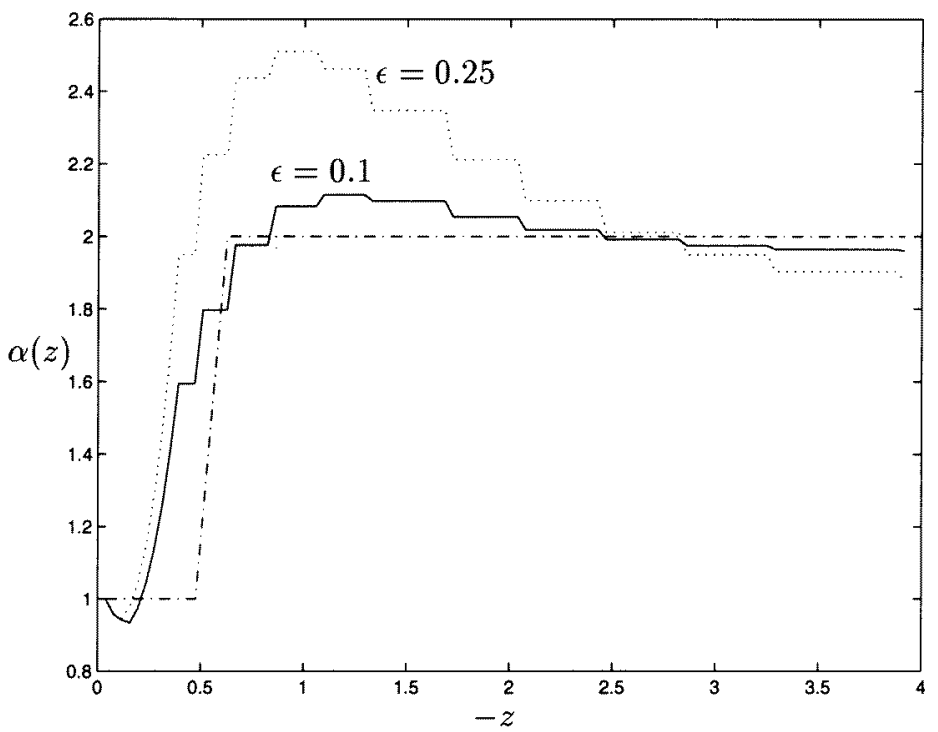

Figure 17. Reconstruction of the layered part of the conductivity $\sigma$ given by (5.1). The true conductivity $\alpha$ is shown with the dash-dotted line. The solid line shows the image for a perturbation parameter $\epsilon=0.1$ and the dotted line shows the image for $\epsilon=0.25$.

\section{Summary}

We have introduced an inversion algorithm for tomographic imaging of one-dimensional media. The algorithm is based on a multiscattering series expansion of the Green function of the layered media. The multiscattering series has a probabilistic interpretation in terms of random walks in the medium. Each term in the series is interpreted as the contribution of paths that reflect multiple times at interfaces separating various layers in the medium. Furthermore, the multiscattering series can be seen as an expansion of the Green function in powers of the reflection coefficients $D_{j}$ that are related to the conductivity of the layered medium through $D_{j}=\frac{\sigma_{j}-\sigma_{j+1}}{\sigma_{j}+\sigma_{j+1}}$. The Born series, that is known to converge only for small contrasts, is shown to be inferior to the multiscattering series that converges unconditionally. In inversion, we estimate the reflection coefficients $D_{j}$ of the layers iteratively, from the multiscattering series, where at each step we consider terms with higher powers of the unknown $D_{j}$. The estimation of the reflection coefficients at each step is done by solving a nonlinear least-squares problem via Newton's method, where the initial guess is the solution obtained at the previous step. In the first step, the problem is linear and we solve it with singular value decomposition.

The location of the layers of constant conductivity is unknown in inversion, but a discretization in depth must be done for numerical computations. Thus, we introduce an a priori, nonuniform discretization of the medium, that takes into account the noise level, the limited resolution and depth of penetration associated with each frequency in the data. For this discretization, we assume $\sigma$ to be constant between two mesh points.

The performance of the inversion algorithm is illustrated through numerical experiments. The images identify correctly the location of the discontinuities in $\sigma$ even when the mesh points of the a priori discretization in depth do not coincide with the location of the layers of the true conductivity. The images also give a good estimate of the value of the conductivity in the medium. Furthermore, the algorithm is shown to be stable and give good images even for 
noisy data.

Our numerical experiments consider media with contrasts ranging from low to intermediate. We show that the algorithm can resolve correctly media with contrasts as high as $300 \%$ in just three steps (i.e. the first three terms in the multiscattering series). Furthermore, Newton's iteration used to solve the nonlinear least-squares problem at each step of the algorithm converges very fast due to the good initial guess provided by the solution of the least-squares problem at the previous step. For low contrasts of about $15 \%$ we show that the algorithm converges in one iteration. However, as the contrast increases, one has to use more and more terms in the multiscattering series (multiple reflections at the interfaces in the medium) and the calculations can become quite complicated. Furthermore, the first step of the algorithm that takes into account only paths that reflect once at some interface, might give an answer that is a poor guess for Newton's method required to solve the nonlinear least-squares problems at later steps. Thus, as expected, the problem becomes more difficult if the contrast is very high but is easily solved for intermediate and low contrasts.

\section{Acknowledgments}

We thank Professor W Symes for several useful discussions and for informing us on the layer stripping work for the Helmoltz equation. We also thank Dr J Berryman for very useful information on the issue of data measurement precision and Professor J B Keller for pointing us to $[22,7,8]$. The work of L Borcea was partially supported by the National Science Foundation under grant number DMS-9627407.

\section{Appendix. Calculation of the probability of the reflected paths in a medium with one interface}

We calculate the probability of the reflected path given by formula (2.18). The integrals in $\alpha$ and $\beta$ are straightforward and the result is

$$
\begin{aligned}
g_{n}^{(r)}\left(\boldsymbol{x}, \boldsymbol{x}^{\prime}\right)= & D \frac{h^{2}}{6 \sigma^{+}} \frac{\left(z+z^{\prime}-2 \xi\right)}{n}\left(\frac{3}{2 \pi n h^{2}}\right) \mathrm{e}^{-\frac{3}{2 n h^{2}}\left[\left(x-x^{\prime}\right)^{2}+\left(y-y^{\prime}\right)^{2}\right]} \\
& \times \sum_{p=0}^{n} \sqrt{\frac{3}{2 \pi p h^{2}}} \mathrm{e}^{-\frac{3}{2 p h^{2}}\left(z^{\prime}-\xi\right)^{2}} \sqrt{\frac{3}{2 \pi(n-p) h^{2}}} \mathrm{e}^{-\frac{3}{2(n-p) h^{2}}(z-\xi)^{2}} .
\end{aligned}
$$

We define

$$
\begin{aligned}
& t=n h \\
& \tau_{p}=p h \\
& \Delta \tau=\tau_{p+1}-\tau_{p}=h \\
& \lambda_{p}=\frac{p}{n} \in[0,1]
\end{aligned}
$$

and observe that because $h \rightarrow 0$, the sum in (A.1) is a Riemann sum. Thus, we have

$g_{n}^{(r)}\left(\boldsymbol{x}, \boldsymbol{x}^{\prime}\right)=\frac{D}{6 \sigma^{+}} \frac{\left(z+z^{\prime}-2 \xi\right)}{n}\left(\frac{3}{2 \pi t h}\right) \mathrm{e}^{-\frac{3}{2 t h}\left[\left(x-x^{\prime}\right)^{2}+\left(y-y^{\prime}\right)^{2}\right]} J\left(t, z, z^{\prime}, \xi\right)$,

where

$$
J\left(t, z, z^{\prime}, \xi\right)=\frac{3}{2 \pi} \int_{0}^{t} \frac{1}{\sqrt{\tau(t-\tau)}} \mathrm{e}^{-\frac{3}{2 \tau h}\left(z^{\prime}-\xi\right)^{2}-\frac{3}{2(t-\tau) h}(z-\xi)^{2}} \mathrm{~d} \tau .
$$


In the limit $h \rightarrow 0$ we have that $\frac{\left(z^{\prime}-\xi\right)^{2}}{h}$ and $\frac{(z-\xi)^{2}}{h}$ are large and so the integral in (A.4) is of Laplace type [4]. With the change of variables

$\tau=\lambda t=\frac{a^{2}}{a^{2}+\eta^{2}} t, \quad$ where $\quad \eta \in[0, \infty)$ and $a=\sqrt{\frac{3}{2 t h}}\left(z^{\prime}-\xi\right)$.

and from the Watson lemma [4] we obtain

$$
\begin{aligned}
J\left(t, z, z^{\prime}, \xi\right) & =\frac{3 a}{\pi} \exp \left(-a^{2}-a^{2}\left(\frac{z-\xi}{z^{\prime}-\xi}\right)^{2}\right) \int_{0}^{\infty} \frac{1}{a^{2}+\eta^{2}} \exp \left(-\eta^{2}-\frac{a^{4}\left(\frac{z-\xi}{z^{\prime}-\xi}\right)^{2}}{\eta^{2}}\right) \mathrm{d} \eta \\
& =\frac{3}{\pi a\left(1+\frac{z-\xi}{z^{\prime}-\xi}\right)}\left\{\int_{0}^{\infty}[1+\mathrm{O}(h)] \exp \left(-\eta^{2}-\frac{a^{4}\left(\frac{z-\xi}{z^{\prime}-\xi}\right)^{2}}{\eta^{2}}\right) \mathrm{d} \eta\right\} .
\end{aligned}
$$

The integral on the right-hand side of equation (A.6) is found in $[1,5]$ and the result is

$$
J\left(t, z, z^{\prime}, \xi\right)=\frac{t h}{\left(z+z^{\prime}-2 \xi\right)} \sqrt{\frac{3}{2 \pi t h}} \exp \left(-\frac{3}{2 t h}\left(z+z^{\prime}-2 \xi\right)^{2}\right)[1+\mathrm{O}(h)] .
$$

The probability of the reflected path is obtained from (A.3) and (A.7):

$$
\begin{aligned}
g_{n}^{(r)}\left(\boldsymbol{x}, \boldsymbol{x}^{\prime}\right)= & D \frac{h^{2}}{6 \sigma^{+}}\left(\frac{3}{2 \pi n h^{2}}\right)^{\frac{3}{2}} \exp \left\{-\frac{3}{2 n h^{2}}\left[\left(x-x^{\prime}\right)^{2}+\left(y-y^{\prime}\right)^{2}+\left(2 \xi-z-z^{\prime}\right)^{2}\right]\right\} \\
& \times[1+\mathrm{O}(h)] .
\end{aligned}
$$

Finally, we calculate the contribution of the reflected path to the Green function

$$
\begin{aligned}
G^{(r)}\left(\boldsymbol{x}, \boldsymbol{x}^{\prime}\right)= & \lim _{h \rightarrow 0} \sum_{n=0}^{\infty} D \frac{h^{2}}{6 \sigma^{+}}\left(\frac{3}{2 \pi n h^{2}}\right)^{\frac{3}{2}} \\
& \times \exp \left\{-\frac{3}{2 n h^{2}}\left[\left(x-x^{\prime}\right)^{2}+\left(y-y^{\prime}\right)^{2}+\left(2 \xi-z-z^{\prime}\right)^{2}\right]\right\}[1+\mathrm{O}(h)] \\
= & \frac{D}{6 \sigma^{+}} \int_{0}^{\infty}\left(\frac{3}{2 \pi s}\right)^{\frac{3}{2}} \exp \left\{-\frac{3}{2 s}\left[\left(x-x^{\prime}\right)^{2}+\left(y-y^{\prime}\right)^{2}+\left(2 \xi-z-z^{\prime}\right)^{2}\right]\right\} \mathrm{d} s \\
= & \frac{D}{4 \pi \sigma^{+} \sqrt{\left(x-x^{\prime}\right)^{2}+\left(y-y^{\prime}\right)^{2}+\left(2 \xi-z-z^{\prime}\right)^{2}}} .
\end{aligned}
$$

A similar calculation applies to the probability of the transmitted path given by (2.21).

\section{References}

[1] Abramovitz, M and Stegun I 1972 Handbook of Mathematical Functions (New York: Dover)

[2] Allers A and Santosa F 1991 Stability and resolution analysis of a linearized problem in electrical impedance tomography Inverse Problems 7 515-33

[3] Barber D C and Seagar A D 1987 Fast reconstruction of resistance images Clinical Phys. Physiol. Meas. 8 suppl A 47-54

[4] Bender C M and Orszag S A 1978 Advanced Mathematical Methods for Scientists and Engineers (New York: McGraw-Hill)

[5] Beyer W H 1991 CRC Standard Mathematical Tables and Formulae (Boca Raton, FL: Chemical Rubber Company)

[6] Berryman J G 1994 Tomographic resolution without singular value decomposition Proc. Conf. Cornelius Lanczos International Centenary (North Carolina State University, Raleigh, NC, December 12-17, 1993 ed J D Brown et al (Philadelphia, PA: SIAM) pp 297-9

[7] Bremmer H 1949 The propagation of electromagnetic waves through a stratified medium and its WKB approximation for oblique incidence Physica 15 536-608 
[8] Bremmer H 1951 The WKB approximation as the first term of a geometric-optical series Commun. Pure Appl. Math. 4 105-15

[9] Bruckstein A M and Kailath T 1987 Inverse scattering for discrete transmission-line models SIAM Rev. 29 359-89

[10] Chandrasekhar S 1943 Stochastic problems in physics and astronomy Rev. Mod. Phys. 15 1-89

[11] Chen S and Rokhlin V 1992 On the inverse scattering problem for the Helmholtz equation in one dimension Inverse Problems 8 365-91

[12] Cheney M and Isaacson D 1992 Distinguishability in impedance imaging IEEE Trans. Biomed. Eng. 39

[13] Cherkaeva E and Tripp A 1996 Inverse conductivity problem for noisy measurements Inverse Problems 12 $869-83$

[14] Curtis E B and Morrow J A 1990 Determining the resistors in a network SIAM J. Appl. Math. 50 918-30

[15] Dobson D C and Santosa F 1994 Resolution and stability analysis of an inverse problem in electrical impedance tomography: dependence on the input current patterns SIAM J. Appl. Math. 54 1542-60

[16] Feynman R P 1972 Statistical Mechanics. A set of lectures (New York: Benjamin)

[17] Golub G H and Van Loan C F 1989 Matrix Computations (Baltimore, MD: John Hopkins University Press)

[18] Houa P, Woo J E, Webster J G and Tompkins W J 1992 Improved methods to determine optimal currents in electrical impedance tomography IEEE Trans. Med. Imag. 11 488-95

[19] Isaacson D 1986 Distinguishability of conductivities by electric current computed tomography IEEE Trans. Med. Imag. 5 91-5

[20] Kac M 1959 Probability and Related Topics in Physical Sciences (London: Interscience)

[21] Keller G V and Frischknecht F C 1966 Electrical Methods in Geophysical Prospecting (Oxford: Pergamon) pp 304-5

[22] Keller H B and Keller J B 1962 Exponential-like solutions of systems of linear ordinary differential equations J. Soc. Indust. Appl. Math. $10246-59$

[23] Kohn R and Vogelius M 1985 Determining conductivity by boundary measurements Commun. Pure Appl. Math. 38 643-67

[24] Kohn R and Vogelius M 1987 Relaxation of a variational method for impedance computed tomography Commun. Pure Appl. Math. XL 745-77

[25] Kozulin Y N 1962 A reflection method for computing the electromagnetic field above horizontal lamellar structures Izv. Geo. Ser. 3 432-43

[26] Newman G A and Hohmann G W 1988 Transient electromagnetic responses of high-contrast prisms in a layered Earth Geophysics $\mathbf{5 3}$ 691-706

[27] Ramirez A, Daily W, Binley A, LaBrecque D and Roelant D 1996 Detection of leaks in underground storage tanks using electrical resistance methods J. Environ. Eng. Geophys. 1 189-203

[28] Schulman L S 1981 Techniques and Applications of Path Integration (New York: Wiley)

[29] Somersalo E, Cheney M, Isaacson D and Isaacson E 1991 Layer stripping: a direct numerical method for impedance imaging Inverse Problems 7 899-926

[30] Sylvester J, Winebrenner D and Gylys-Colwell F 1996 Layer stripping for the Helmholtz equation SIAM J. Appl. Math. 56 736-54

[31] Wexler A, Fry B and Neuman M 1985 Impedance-computed tomography algorithm and system Appl. Opt. 24 $3985-2$

[32] Wiegel F W 1986 Introduction to Path-Integral Methods in Physics and Polymer Science (Philadelphia, PA: Word Scientific)

[33] Yorkey T J, Webster J G and Tompkins W J 1987 Comparing reconstruction algorithms for electrical impedance tomography IEEE Trans. Biomed. Engng 34 843-52 